\title{
\begin{tabular}{l|l} 
Mitraries & DSpace@MIT
\end{tabular}
}

\author{
MIT Open Access Articles
}

Search for anomalous production of events with two photons and additional energetic objects at CDF

The MIT Faculty has made this article openly available. Please share how this access benefits you. Your story matters.

Citation: Aaltonen, T. et al. "Search for anomalous production of events with two photons and additional energetic objects at CDF." Physical Review D 82.5 (2010): n. pag. (C) 2011 by The American Physical Society

As Published: http://dx.doi.org/10.1103/PhysRevD.82.052005

Publisher: American Physical Society

Persistent URL: http://hdl.handle.net/1721.1/60452

Version: Final published version: final published article, as it appeared in a journal, conference proceedings, or other formally published context

Terms of Use: Article is made available in accordance with the publisher's policy and may be subject to US copyright law. Please refer to the publisher's site for terms of use. 


\section{Search for anomalous production of events with two photons and additional energetic objects at CDF}

T. Aaltonen, ${ }^{24}$ J. Adelman, ${ }^{14}$ B. Álvarez González, ${ }^{12, \mathrm{x}}$ S. Amerio, ${ }^{44 \mathrm{~b}, 44 \mathrm{a}}$ D. Amidei, ${ }^{35}$ A. Anastassov, ${ }^{39}$ A. Annovi, ${ }^{20}$ J. Antos,${ }^{15}$ G. Apollinari, ${ }^{18}$ A. Apresyan, ${ }^{49}$ T. Arisawa, ${ }^{58}$ A. Artikov, ${ }^{16}$ J. Asaadi, ${ }^{54}$ W. Ashmanskas,${ }^{18}$ A. Attal, ${ }^{4}$ A. Aurisano, ${ }^{54}$ F. Azfar, ${ }^{43}$ W. Badgett, ${ }^{18}$ A. Barbaro-Galtieri, ${ }^{29}$ V. E. Barnes,${ }^{49}$ B. A. Barnett, ${ }^{26}$ P. Barria, ${ }^{47 c, 47 a}$ P. Bartos,${ }^{15}$ G. Bauer, ${ }^{33}$ P.-H. Beauchemin, ${ }^{34}$ F. Bedeschi, ${ }^{47 a}$ D. Beecher, ${ }^{31}$ S. Behari, ${ }^{26}$ G. Bellettini, ${ }^{47 b, 47 a}$ J. Bellinger, ${ }^{60}$ D. Benjamin, ${ }^{17}$ A. Beretvas, ${ }^{18}$ A. Bhatti, ${ }^{51}$ M. Binkley, ${ }^{18}$ D. Bisello, ${ }^{44 b, 44 a}$ I. Bizjak, ${ }^{31, e e}$ R. E. Blair, ${ }^{2}$ C. Blocker, ${ }^{7}$ B. Blumenfeld, ${ }^{26}$ A. Bocci, ${ }^{17}$ A. Bodek,${ }^{50}$ V. Boisvert, ${ }^{50}$ D. Bortoletto, ${ }^{49}$ J. Boudreau, ${ }^{48}$ A. Boveia, ${ }^{11}$ B. Brau, ${ }^{11, \mathrm{c}}$ A. Bridgeman, ${ }^{25}$ L. Brigliadori, ${ }^{6 b, 6 a}$ C. Bromberg, ${ }^{36}$ E. Brubaker, ${ }^{14}$ J. Budagov, ${ }^{16}$ H. S. Budd, ${ }^{50}$ S. Budd,${ }^{25}$ K. Burkett, ${ }^{18}$ G. Busetto, ${ }^{44 b, 44 a}$ P. Bussey, ${ }^{22}$ A. Buzatu, ${ }^{34}$ K. L. Byrum, ${ }^{2}$ S. Cabrera, ${ }^{17, z}$ C. Calancha, ${ }^{32}$ S. Camarda, ${ }^{4}$ M. Campanelli, ${ }^{36}$ M. Campbell, ${ }^{35}$ F. Canelli, ${ }^{14,18}$ A. Canepa, ${ }^{46}$ B. Carls ${ }^{25}$ D. Carlsmith, ${ }^{60}$ R. Carosi, ${ }^{47 a}$ S. Carrillo, ${ }^{19, p}$ S. Carron, ${ }^{18}$ B. Casal, ${ }^{12}$ M. Casarsa, ${ }^{18}$ A. Castro, ${ }^{6 b, 6 a}$ P. Catastini, ${ }^{47 c, 47 a}$ D. Cauz,${ }^{55 a}$ V. Cavaliere,${ }^{47 c, 47 a}$ M. Cavalli-Sforza, ${ }^{4}$ A. Cerri, ${ }^{29}$ L. Cerrito, ${ }^{31, \mathrm{~s}}$ S. H. Chang, ${ }^{28}$ Y. C. Chen, ${ }^{1}$ M. Chertok, ${ }^{8}$ G. Chiarelli, ${ }^{47 a}$ G. Chlachidze, ${ }^{18}$ F. Chlebana, ${ }^{18}$ K. Cho, ${ }^{28}$ D. Chokheli, ${ }^{16}$ J. P. Chou, ${ }^{23}$ K. Chung, ${ }^{18, q}$ W. H. Chung, ${ }^{60}$ Y. S. Chung, ${ }^{50}$ T. Chwalek, ${ }^{27}$ C. I. Ciobanu, ${ }^{45}$ M. A. Ciocci, ${ }^{47 c, 47 a}$ A. Clark, ${ }^{21}$ D. Clark, ${ }^{7}$ G. Compostella, ${ }^{44 a}$ M. E. Convery, ${ }^{18}$ J. Conway, ${ }^{8}$ M. Corbo, ${ }^{45}$ M. Cordelli, ${ }^{20}$ C. A. Cox,${ }^{8}$ D. J. Cox,${ }^{8}$ F. Crescioli, ${ }^{47 b, 47 a}$ C. Cuenca Almenar, ${ }^{61}$ J. Cuevas, ${ }^{12, x}$ R. Culbertson, ${ }^{18}$ J. C. Cully, ${ }^{35}$

D. Dagenhart, ${ }^{18}$ M. Datta, ${ }^{18}$ T. Davies, ${ }^{22}$ P. de Barbaro, ${ }^{50}$ S. De Cecco,${ }^{52 a}$ A. Deisher,${ }^{29}$ G. De Lorenzo,${ }^{4}$

M. Dell'Orso, ${ }^{47 b, 47 a}$ C. Deluca, ${ }^{4}$ L. Demortier, ${ }^{51}$ J. Deng, ${ }^{17, h}$ M. Deninno, ${ }^{6 a}$ M. d'Errico, ${ }^{44 b, 44 a}$ A. Di Canto, ${ }^{47 b, 47 a}$ G. P. di Giovanni ${ }^{45}$ B. Di Ruzza, ${ }^{47 a}$ J. R. Dittmann, ${ }^{5}$ M. D'Onofrio, ${ }^{4}$ S. Donati,${ }^{47 b, 47 a}$ P. Dong, ${ }^{18}$ T. Dorigo, ${ }^{44 a}$ S. Dube, ${ }^{53}$

K. Ebina ${ }^{58}$ A. Elagin,${ }^{54}$ R. Erbacher, ${ }^{8}$ D. Errede,${ }^{25}$ S. Errede, ${ }^{25}$ N. Ershaidat,${ }^{45, d d}$ R. Eusebi,${ }^{54}$ H. C. Fang, ${ }^{29}$

S. Farrington, ${ }^{43}$ W. T. Fedorko, ${ }^{14}$ R. G. Feild, ${ }^{61}$ M. Feindt, ${ }^{27}$ J. P. Fernandez,${ }^{32}$ C. Ferrazza, ${ }^{47 d, 47 a}$ R. Field, ${ }^{19}$

G. Flanagan ${ }^{49, u}$ R. Forrest,${ }^{8}$ M. J. Frank, ${ }^{5}$ M. Franklin, ${ }^{23}$ J. C. Freeman, ${ }^{18}$ I. Furic, ${ }^{19}$ M. Gallinaro, ${ }^{51}$ J. Galyardt, ${ }^{13}$ F. Garberson, ${ }^{11}$ J. E. Garcia, ${ }^{21}$ A. F. Garfinkel, ${ }^{49}$ P. Garosi, ${ }^{47 c, 47 a}$ H. Gerberich, ${ }^{25}$ D. Gerdes,${ }^{35}$ A. Gessler, ${ }^{27}$ S. Giagu, ${ }^{52 b, 52 a}$ V. Giakoumopoulou, ${ }^{3}$ P. Giannetti, ${ }^{47 a}$ K. Gibson, ${ }^{48}$ J. L. Gimmell, ${ }^{50}$ C. M. Ginsburg, ${ }^{18}$ N. Giokaris, ${ }^{3}$ M. Giordani, ${ }^{55 b, 55 a}$ P. Giromini, ${ }^{20}$ M. Giunta, ${ }^{47 a}$ G. Giurgiu, ${ }^{26}$ V. Glagolev, ${ }^{16}$ D. Glenzinski, ${ }^{18}$ M. Gold,${ }^{38}$ N. Goldschmidt,${ }^{19}$ A. Golossanov, ${ }^{18}$

G. Gomez, ${ }^{12}$ G. Gomez-Ceballos, ${ }^{33}$ M. Goncharov, ${ }^{33}$ O. González, ${ }^{32}$ I. Gorelov, ${ }^{38}$ A. T. Goshaw, ${ }^{17}$ K. Goulianos, ${ }^{51}$

A. Gresele, ${ }^{44 b, 44 a}$ S. Grinstein, ${ }^{4}$ C. Grosso-Pilcher, ${ }^{14}$ R. C. Group,${ }^{18}$ U. Grundler, ${ }^{25}$ J. Guimaraes da Costa, ${ }^{23}$

Z. Gunay-Unalan, ${ }^{36}$ C. Haber, ${ }^{29}$ S. R. Hahn, ${ }^{18}$ E. Halkiadakis, ${ }^{53}$ B.-Y. Han,${ }^{50}$ J. Y. Han, ${ }^{50}$ F. Happacher, ${ }^{20}$ K. Hara, ${ }^{56}$

D. Hare ${ }^{53}$ M. Hare ${ }^{57}$ R. F. Harr, ${ }^{59}$ M. Hartz, ${ }^{48}$ K. Hatakeyama, ${ }^{5}$ C. Hays,${ }^{43}$ M. Heck, ${ }^{27}$ J. Heinrich,${ }^{46}$ M. Herndon, ${ }^{60}$

J. Heuser, ${ }^{27}$ S. Hewamanage, ${ }^{5}$ D. Hidas,${ }^{53}$ C. S. Hill, ${ }^{11, \mathrm{e}}$ D. Hirschbuehl,${ }^{27}$ A. Hocker, ${ }^{18}$ S. Hou, ${ }^{1}$ M. Houlden, ${ }^{30}$

S.-C. Hsu, ${ }^{29}$ R. E. Hughes ${ }^{40}$ M. Hurwitz, ${ }^{14}$ U. Husemann, ${ }^{61}$ M. Hussein, ${ }^{36}$ J. Huston, ${ }^{36}$ J. Incandela, ${ }^{11}$ G. Introzzi, ${ }^{47 a}$ M. Iori, ${ }^{52 b, 52 a}$ A. Ivanov, ${ }^{8, \mathrm{r}}$ E. James, ${ }^{18}$ D. Jang, ${ }^{13}$ B. Jayatilaka, ${ }^{17}$ E. J. Jeon, ${ }^{28}$ M. K. Jha ${ }^{6 a}$ S. Jindariani, ${ }^{18}$ W. Johnson, ${ }^{8}$ M. Jones, ${ }^{49}$ K. K. Joo, ${ }^{28}$ S. Y. Jun, ${ }^{13}$ J. E. Jung, ${ }^{28}$ T. R. Junk, ${ }^{18}$ T. Kamon,${ }^{54}$ D. Kar, ${ }^{19}$ P. E. Karchin, ${ }^{59}$ Y. Kato, ${ }^{42, o}$ R. Kephart, ${ }^{18}$ W. Ketchum, ${ }^{14}$ J. Keung, ${ }^{46}$ V. Khotilovich, ${ }^{54}$ B. Kilminster, ${ }^{18}$ D. H. Kim, ${ }^{28}$ H. S. Kim, ${ }^{28}$ H. W. Kim, ${ }^{28}$ J. E. Kim, ${ }^{28}$ M. J. Kim, ${ }^{20}$ S. B. Kim, ${ }^{28}$ S. H. Kim, ${ }^{56}$ Y. K. Kim, ${ }^{14}$ N. Kimura, ${ }^{58}$ L. Kirsch, ${ }^{7}$ S. Klimenko, ${ }^{19}$ K. Kondo, ${ }^{58}$ D. J. Kong, ${ }^{28}$ J. Konigsberg, ${ }^{19}$ A. Korytov, ${ }^{19}$ A. V. Kotwal, ${ }^{17}$ M. Kreps,${ }^{27}$ J. Kroll, ${ }^{46}$ D. Krop, ${ }^{14}$ N. Krumnack,${ }^{5}$ M. Kruse, ${ }^{17}$ V. Krutelyov, ${ }^{11}$ T. Kuhr, ${ }^{27}$ N. P. Kulkarni,${ }^{59}$ M. Kurata, ${ }^{56}$ S. Kwang, ${ }^{14}$ A. T. Laasanen, ${ }^{49}$ S. Lami, ${ }^{47 a}$ S. Lammel, ${ }^{18}$ M. Lancaster, ${ }^{31}$ R. L. Lander, ${ }^{8}$ K. Lannon, ${ }^{40, w}$ A. Lath, ${ }^{53}$ G. Latino, ${ }^{47 c, 47 a}$ I. Lazzizzera, ${ }^{44 b, 44 a}$ T. LeCompte, ${ }^{2}$ E. Lee, ${ }^{54}$ H. S. Lee, ${ }^{14}$ J. S. Lee ${ }^{28}$ S. W. Lee ${ }^{54, y}$ S. Leone, ${ }^{47 a}$ J. D. Lewis, ${ }^{18}$ C.-J. Lin, ${ }^{29}$ J. Linacre, ${ }^{43}$ M. Lindgren, ${ }^{18}$ E. Lipeles, ${ }^{46}$ A. Lister, ${ }^{21}$ D. O. Litvintsev, ${ }^{18}$ C. Liu,${ }^{48}$ T. Liu, ${ }^{18}$ N. S. Lockyer, ${ }^{46}$ A. Loginov, ${ }^{61}$ L. Lovas, ${ }^{15}$ D. Lucchesi, ${ }^{44 b, 44 a}$ J. Lueck. ${ }^{27}$ P. Lujan, ${ }^{29}$ P. Lukens, ${ }^{18}$ G. Lungu, ${ }^{51}$ J. Lys,${ }^{29}$ R. Lysak, ${ }^{15}$ D. MacQueen,${ }^{34}$ R. Madrak,${ }^{18}$ K. Maeshima,${ }^{18}$ K. Makhoul, ${ }^{33}$ P. Maksimovic, ${ }^{26}$ S. Malde ${ }^{43}$ S. Malik, ${ }^{31}$ G. Manca, ${ }^{30,9}$ A. Manousakis-Katsikakis, ${ }^{3}$ F. Margaroli, ${ }^{49}$ C. Marino, ${ }^{27}$

C. P. Marino, ${ }^{25}$ A. Martin, ${ }^{61}$ V. Martin, ${ }^{22, m}$ M. Martínez, ${ }^{4}$ R. Martínez-Ballarín, ${ }^{32}$ P. Mastrandrea,${ }^{52 a}$ M. Mathis, ${ }^{26}$ M. E. Mattson, ${ }^{59}$ P. Mazzanti, ${ }^{6 a}$ K. S. McFarland,${ }^{50}$ P. McIntyre, ${ }^{54}$ R. McNulty, ${ }^{30,1}$ A. Mehta, ${ }^{30}$ P. Mehtala, ${ }^{24}$

A. Menzione, ${ }^{47 a}$ C. Mesropian, ${ }^{51}$ T. Miao, ${ }^{18}$ D. Mietlicki, ${ }^{35}$ N. Miladinovic, ${ }^{7}$ R. Miller, ${ }^{36}$ C. Mills, ${ }^{23}$ M. Milnik, ${ }^{27}$ A. Mitra, ${ }^{1}$ G. Mitselmakher, ${ }^{19}$ H. Miyake, ${ }^{56}$ S. Moed, ${ }^{23}$ N. Moggi, ${ }^{6 a}$ M. N. Mondragon, ${ }^{18, p}$ C. S. Moon, ${ }^{28}$ R. Moore,${ }^{18}$ M. J. Morello, ${ }^{47 a}$ J. Morlock, ${ }^{27}$ P. Movilla Fernandez, ${ }^{18}$ J. Mülmenstädt, ${ }^{29}$ A. Mukherjee, ${ }^{18}$ Th. Muller,${ }^{27}$ P. Murat,${ }^{18}$ M. Mussini, ${ }^{6 b, 6 a}$ J. Nachtman, ${ }^{18, q}$ Y. Nagai, ${ }^{56}$ J. Naganoma, ${ }^{56}$ K. Nakamura, ${ }^{56}$ I. Nakano, ${ }^{41}$ A. Napier,${ }^{57}$ J. Nett, ${ }^{60}$ C. Neu, ${ }^{46, b b}$ M. S. Neubauer, ${ }^{25}$ S. Neubauer, ${ }^{27}$ J. Nielsen, ${ }^{29, i}$ L. Nodulman, ${ }^{2}$ M. Norman, ${ }^{10}$ O. Norniella,${ }^{25}$ E. Nurse,${ }^{31}$ L. Oakes, ${ }^{43}$ S. H. Oh, ${ }^{17}$ Y. D. Oh, ${ }^{28}$ I. Oksuzian, ${ }^{19}$ T. Okusawa, ${ }^{42}$ R. Orava,${ }^{24}$ K. Osterberg, ${ }^{24}$ S. Pagan Griso, ${ }^{44 b, 44 a}$ 
C. Pagliarone, ${ }^{55 a}$ E. Palencia, ${ }^{18}$ V. Papadimitriou, ${ }^{18}$ A. Papaikonomou,${ }^{27}$ A. A. Paramanov, ${ }^{2}$ B. Parks, ${ }^{40}$ S. Pashapour, ${ }^{34}$ J. Patrick, ${ }^{18}$ G. Pauletta, ${ }^{55 b, 55 a}$ M. Paulini, ${ }^{13}$ C. Paus,${ }^{33}$ T. Peiffer, ${ }^{27}$ D. E. Pellett, ${ }^{8}$ A. Penzo, ${ }^{55 a}$ T. I. Phillips,${ }^{17}$ G. Piacentino, ${ }^{47 a}$ E. Pianori, ${ }^{46}$ L. Pinera, ${ }^{19}$ K. Pitts, ${ }^{25}$ C. Plager, ${ }^{9}$ L. Pondrom,${ }^{60}$ K. Potamianos, ${ }^{49}$ O. Poukhov, ${ }^{16, a}$ F. Prokoshin, ${ }^{16, a a}$ A. Pronko, ${ }^{18}$ F. Ptohos, ${ }^{18, k}$ E. Pueschel, ${ }^{13}$ G. Punzi, ${ }^{47 b, 47 a}$ J. Pursley, ${ }^{60}$ J. Rademacker, ${ }^{43, e}$ A. Rahaman, ${ }^{48}$ V. Ramakrishnan, ${ }^{60}$ N. Ranjan, ${ }^{49}$ I. Redondo, ${ }^{32}$ P. Renton, ${ }^{43}$ M. Renz,${ }^{27}$ M. Rescigno, ${ }^{52 a}$ S. Richter, ${ }^{27}$ F. Rimondi, ${ }^{6 b, 6 a}$ L. Ristori, ${ }^{47 a}$ A. Robson, ${ }^{22}$ T. Rodrigo, ${ }^{12}$ T. Rodriguez,${ }^{46}$ E. Rogers,${ }^{25}$ S. Rolli,${ }^{57}$ R. Roser,${ }^{18}$ M. Rossi,${ }^{55 a}$ R. Rossin, ${ }^{11}$ P. Roy ${ }^{34}$ A. Ruiz, ${ }^{12}$ J. Russ, ${ }^{13}$ V. Rusu, ${ }^{18}$ B. Rutherford,${ }^{18}$ H. Saarikko, ${ }^{24}$ A. Safonov, ${ }^{54}$ W. K. Sakumoto, ${ }^{50}$ L. Santi, ${ }^{55 b, 55 a}$ L. Sartori, ${ }^{47 a}$ K. Sato, ${ }^{56}$ A. Savoy-Navarro, ${ }^{45}$ P. Schlabach, ${ }^{18}$ A. Schmidt, ${ }^{27}$ E. E. Schmidt, ${ }^{18}$ M. A. Schmidt, ${ }^{14}$ M. P Schmidt, ${ }^{61, a}$ M. Schmitt, ${ }^{39}$ T. Schwarz, ${ }^{8}$ L. Scodellaro, ${ }^{12}$ A. Scribano, ${ }^{47 c, 47 a}$ F. Scuri, ${ }^{47 a}$ A. Sedov, ${ }^{49}$ S. Seidel, ${ }^{38}$ Y. Seiya, ${ }^{42}$ A. Semenov, ${ }^{16}$ L. Sexton-Kennedy, ${ }^{18}$ F. Sforza, ${ }^{47 b, 47 a}$ A. Sfyrla ${ }^{25}$ S. Z. Shalhout, ${ }^{59}$ T. Shears, ${ }^{30}$ P. F. Shepard ${ }^{48}$ M. Shimojima, ${ }^{56, v}$ S. Shiraishi, ${ }^{14}$ M. Shochet, ${ }^{14}$ Y. Shon,${ }^{60}$ I. Shreyber,${ }^{37}$ A. Simonenko, ${ }^{16}$ P. Sinervo, ${ }^{34}$ A. Sisakyan, ${ }^{16}$ A. J. Slaughter, ${ }^{18}$ J. Slaunwhite, ${ }^{40}$ K. Sliwa,${ }^{57}$ J. R. Smith,${ }^{8}$ F. D. Snider, ${ }^{18}$ R. Snihur, ${ }^{34}$ A. Soha, ${ }^{18}$ S. Somalwar, ${ }^{53}$ V. Sorin, ${ }^{4}$ P. Squillacioti, ${ }^{47 c, 47 a}$ M. Stanitzki, ${ }^{61}$ R. St. Denis, ${ }^{22}$ B. Stelzer, ${ }^{34}$ O. Stelzer-Chilton, ${ }^{34}$ D. Stentz, ${ }^{39}$ J. Strologas, ${ }^{38}$ G. L. Strycker, ${ }^{35}$ J. S. Suh, ${ }^{28}$ A. Sukhanov, ${ }^{19}$ I. Suslov, ${ }^{16}$ A. Taffard, ${ }^{25, h}$ R. Takashima, ${ }^{41}$ Y. Takeuchi, ${ }^{56}$ R. Tanaka, ${ }^{41}$ J. Tang, ${ }^{14}$ M. Tecchio, ${ }^{35}$ P. K. Teng, ${ }^{1}$ J. Thom,,${ }^{18, j}$ J. Thome, ${ }^{13}$ G. A. Thompson, ${ }^{25}$ E. Thomson, ${ }^{46}$ P. Tipton, ${ }^{61}$ P. Ttito-Guzmán, ${ }^{32} \mathrm{~S}$. Tkaczyk, ${ }^{18} \mathrm{D}$. Toback,${ }^{54} \mathrm{~S}$. Tokar, ${ }^{15} \mathrm{~K}$. Tollefson, ${ }^{36} \mathrm{~T}$. Tomura, ${ }^{56} \mathrm{D}$. Tonelli, ${ }^{18} \mathrm{~S}$. Torre,${ }^{20}$ D. Torretta, ${ }^{18}$ P. Totaro, ${ }^{55 b, 55 a}$ S. Tourneur, ${ }^{45}$ M. Trovato, ${ }^{47 d, 47 a}$ S.-Y. Tsai, ${ }^{1}$ Y. Tu, ${ }^{46}$ N. Turini,${ }^{47 c, 47 a}$ F. Ukegawa, ${ }^{56}$ S. Uozumi, ${ }^{28}$ N. van Remortel, ${ }^{24, d}$ A. Varganov, ${ }^{35}$ E. Vataga, ${ }^{47 d, 47 a}$ F. Vázquez, ${ }^{19, p}$ G. Velev ${ }^{18}$ C. Vellidis, ${ }^{3}$ M. Vidal, ${ }^{32}$ I. Vila, ${ }^{12}$ R. Vilar, ${ }^{12}$ M. Vogel,${ }^{38}$ I. Volobouev, ${ }^{29, y}$ G. Volpi,${ }^{47 b, 47 a}$ P. Wagner, ${ }^{46}$ R. G. Wagner, ${ }^{2}$ R. L. Wagner, ${ }^{18}$ W. Wagner, ${ }^{27, c c}$ J. Wagner-Kuhr, ${ }^{27}$ T. Wakisaka, ${ }^{42}$ R. Wallny, ${ }^{9}$ S. M. Wang, ${ }^{1}$ A. Warburton, ${ }^{34}$ D. Waters, ${ }^{31}$ M. Weinberger, ${ }^{54}$ J. Weinelt, ${ }^{27}$ W. C. Wester III, ${ }^{18}$ B. Whitehouse, ${ }^{57}$ D. Whiteson, ${ }^{46, \mathrm{~h}}$ A. B. Wicklund, ${ }^{2}$ E. Wicklund ${ }^{18}$ S. Wilbur, ${ }^{14}$ G. Williams, ${ }^{34}$ H. H. Williams,${ }^{46}$ P. Wilson, ${ }^{18}$ B. L. Winer, ${ }^{40}$ P. Wittich, ${ }^{18, j}$ S. Wolbers,${ }^{18}$ C. Wolfe, ${ }^{14}$ H. Wolfe, ${ }^{40}$ T. Wright,${ }^{35}$ X. Wu, ${ }^{21}$ F. Würthwein, ${ }^{10}$ A. Yagil, ${ }^{10}$ K. Yamamoto, ${ }^{42}$ J. Yamaoka, ${ }^{17}$ U. K. Yang, ${ }^{14, t}$ Y. C. Yang,${ }^{28}$ W. M. Yao, ${ }^{29}$ G. P. Yeh, ${ }^{18}$ K. Yi, ${ }^{18, q}$ J. Yoh, ${ }^{18}$ K. Yorita, ${ }^{58}$ T. Yoshida,${ }^{42, n}$ G. B. Yu, ${ }^{17}$ I. Yu, ${ }^{28}$ S. S. Yu, ${ }^{18}$ J. C. Yun, ${ }^{18}$ A. Zanetti, ${ }^{55 \mathrm{a}}$ Y. Zeng, ${ }^{17} \mathrm{X}$. Zhang, ${ }^{25}$ Y. Zheng,,${ }^{9, \mathrm{f}}$ and S. Zucchelli ${ }^{\mathrm{bb}, 6 \mathrm{a}}$

\title{
$(\mathrm{CDF} \text { Collaboration })^{\mathrm{b}}$
}

\author{
${ }^{1}$ Institute of Physics, Academia Sinica, Taipei, Taiwan 11529, Republic of China \\ ${ }^{2}$ Argonne National Laboratory, Argonne, Illinois 60439, USA \\ ${ }^{3}$ University of Athens, 15771 Athens, Greece \\ ${ }^{4}$ Institut de Fisica d'Altes Energies, Universitat Autonoma de Barcelona, E-08193, Bellaterra (Barcelona), Spain \\ ${ }^{5}$ Baylor University, Waco, Texas 76798, USA \\ ${ }^{6 a}$ Istituto Nazionale di Fisica Nucleare Bologna, I-40127 Bologna, Italy \\ ${ }^{6 \mathrm{~b}}$ University of Bologna, I-40127 Bologna, Italy \\ ${ }^{7}$ Brandeis University, Waltham, Massachusetts 02254, USA \\ ${ }^{8}$ University of California, Davis, Davis, California 95616, USA \\ ${ }^{9}$ University of California, Los Angeles, Los Angeles, California 90024, USA \\ ${ }^{10}$ University of California, San Diego, La Jolla, California 92093, USA \\ ${ }^{11}$ University of California, Santa Barbara, Santa Barbara, California 93106 \\ ${ }^{12}$ Instituto de Fisica de Cantabria, CSIC-University of Cantabria, 39005 Santander, Spain \\ ${ }^{13}$ Carnegie Mellon University, Pittsburgh, Pennsylvania 15213, USA \\ ${ }^{14}$ Enrico Fermi Institute, University of Chicago, Chicago, Illinois 60637, USA \\ ${ }^{15}$ Comenius University, 84248 Bratislava, Slovakia; and Institute of Experimental Physics, \\ 04001 Kosice, Slovakia \\ ${ }^{16}$ Joint Institute for Nuclear Research, RU-141980 Dubna, Russia \\ ${ }^{17}$ Duke University, Durham, North Carolina 27708, USA \\ ${ }^{18}$ Fermi National Accelerator Laboratory, Batavia, Illinois 60510, USA \\ ${ }^{19}$ University of Florida, Gainesville, Florida 32611, USA \\ ${ }^{20}$ Laboratori Nazionali di Frascati, Istituto Nazionale di Fisica Nucleare, I-00044 Frascati, Italy \\ ${ }^{21}$ University of Geneva, CH-1211 Geneva 4, Switzerland \\ ${ }^{22}$ Glasgow University, Glasgow G12 8QQ, United Kingdom \\ ${ }^{23}$ Harvard University, Cambridge, Massachusetts 02138, USA \\ ${ }^{24}$ Division of High Energy Physics, Department of Physics, University of Helsinki and Helsinki Institute of Physics,
} FIN-00014, Helsinki, Finland 


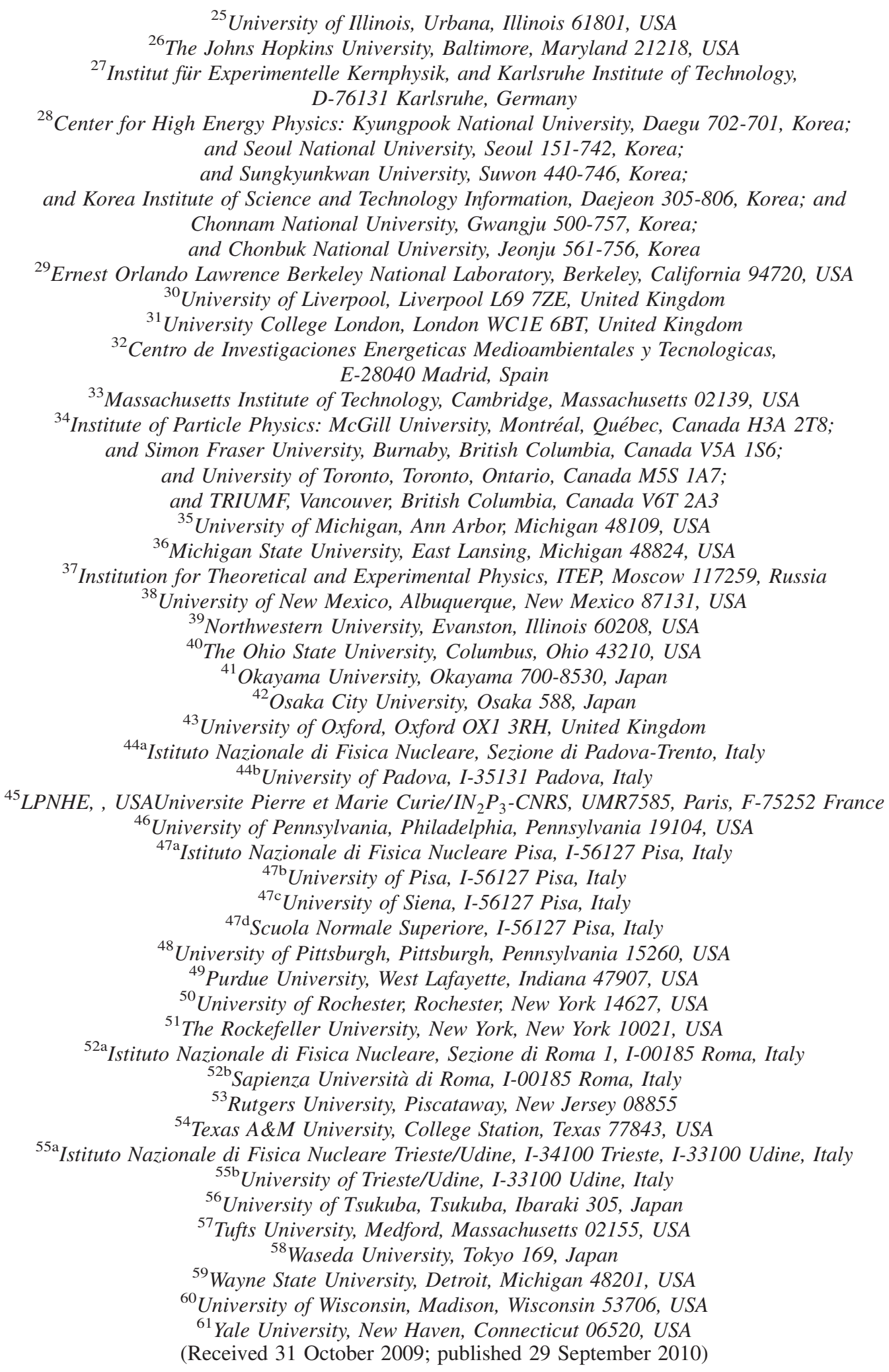

We present results of a search for anomalous production of two photons together with an electron, muon, $\tau$ lepton, missing transverse energy, or jets using $p \bar{p}$ collision data from $1.1-2.0 \mathrm{fb}^{-1}$ of integrated luminosity collected by the Collider Detector at Fermilab (CDF). The event yields and kinematic distributions are examined for signs of new physics without favoring a specific model of new physics. The results are consistent with the standard model expectations. The search employs several new analysis 
techniques that significantly reduce instrumental backgrounds in channels with an electron and missing transverse energy.

\section{INTRODUCTION}

Over the last 20 years, the rapid pace of developments in phenomenology and model-building has left experimentalists at the collider energy frontier with a wide array of new physics scenarios to investigate [1]. We are also assured that the number of models which have not yet been described is large. Since each search requires substantial resources, only a few new physics scenarios can be the focus of dedicated efforts. We address this problem by performing broad searches in available data samples for any discrepancy with the standard model (SM) [2] in event yields or kinematic distributions. While this approach is not optimized for any particular scenario, it could possibly increase the chance of an unpredicted discovery.

In this article we investigate a sample of data collected by the CDF II detector in $p \bar{p}$ collisions at $\sqrt{s}=1.96 \mathrm{TeV}$ at the Fermilab Tevatron. We restrict ourselves to a "baseline" sample with two isolated, central $(0.05<|\eta|<$ 1.05) photons $(\gamma)$ with $E_{T}>13 \mathrm{GeV}$ [3]. We then select subsamples which also contain at least one more energetic, isolated, and well-identified object or where two photons are accompanied by large missing transverse energy $\left(E_{T}\right)$. The additional object may be an electron $(e)$, muon $(\mu)$, or $\tau$ lepton $(\tau)$. The $E_{T}$ is calculated from the imbalance in the energy of visible particles projected to the plane transverse to the beams. The integrated luminosity for each subsample varies from 1.1 to $2.0 \mathrm{fb}^{-1}$.

The $\gamma \gamma+X\left(X=e / \mu, \tau\right.$, and $\left.E_{T}\right)$ signatures are present in many new physics scenarios beyond the SM. Examples include models with the gauge-mediated supersymmetry breaking [4], extended Higgs sector [5], technicolor models [6], fourth generation fermions [7], and theories with large extra dimensions [8].

The CDF collaboration has previously performed a search for anomalous production of two photons and an additional energetic object $\left(E_{T}, e, \mu, \tau, \gamma\right.$, jets, and $b$-quarks) in $85 \mathrm{pb}^{-1}$ of the Tevatron Run I data [9]. Apart from the observation of a single ee $\gamma \gamma E_{T}$ candidate event, the results were consistent with the SM predictions.

\footnotetext{
${ }^{\mathrm{a}}$ Deceased

${ }^{\mathrm{b}}$ With visitors from

${ }^{\mathrm{c}}$ University of Massachusetts Amherst, Amherst, MA 01003, USA

${ }^{\mathrm{d}}$ Universiteit Antwerpen, B-2610 Antwerp, Belgium

${ }^{\mathrm{e}}$ University of Bristol, Bristol BS8 1TL, United Kingdom

${ }^{\mathrm{f}}$ Chinese Academy of Sciences, Beijing 100864, China

${ }^{\mathrm{g}}$ Istituto Nazionale di Fisica Nucleare, Sezione di Cagliari, 09042 Monserrato (Cagliari), Italy

${ }^{\mathrm{h}}$ University of California Irvine, Irvine, CA 92697, USA

${ }^{\mathrm{i}}$ University of California Santa Cruz, Santa Cruz, CA 95064, USA

${ }^{\mathrm{j}}$ Cornell University, Ithaca, NY 14853, USA

${ }^{k}$ University of Cyprus, Nicosia CY-1678, Cyprus

${ }^{1}$ University College Dublin, Dublin 4, Ireland

${ }^{\mathrm{m}}$ University of Edinburgh, Edinburgh EH9 3JZ, United Kingdom

${ }^{\mathrm{n}}$ University of Fukui, Fukui City, Fukui Prefecture, Japan 910-0017

${ }^{\circ}$ Kinki University, Higashi-Osaka City, Japan 577-8502

${ }^{\mathrm{p}}$ Universidad Iberoamericana, Mexico D.F., Mexico

${ }^{\mathrm{q}}$ University of Iowa, Iowa City, IA 52242, USA

${ }^{\mathrm{r}}$ Kansas State University, Manhattan, KS 66506, USA

${ }^{\mathrm{s}}$ Queen Mary, University of London, London, E1 4NS, England

${ }^{\mathrm{t}}$ University of Manchester, Manchester M13 9PL, England

${ }^{u}$ Muons, Inc., Batavia, IL 60510, USA

${ }^{\mathrm{v}}$ Nagasaki Institute of Applied Science, Nagasaki, Japan

${ }^{\mathrm{w}}$ University of Notre Dame, Notre Dame, IN 46556, USA

${ }^{\mathrm{x}}$ University de Oviedo, E-33007 Oviedo, Spain

${ }^{\mathrm{y}}$ Texas Tech University, Lubbock, TX 79609, USA

${ }^{\mathrm{z}}$ IFIC(CSIC-Universitat de Valencia), 56071 Valencia, Spain

${ }^{\text {aa } U n i v e r s i d a d ~ T e c n i c a ~ F e d e r i c o ~ S a n t a ~ M a r i a, ~ 110 v ~ V a l p a r a i s o, ~ C h i l e ~}$

${ }^{\mathrm{bb}}$ University of Virginia, Charlottesville, VA 22906, USA

${ }^{\mathrm{cc}}$ Bergische Universität Wuppertal, 42097 Wuppertal, Germany

${ }^{\mathrm{dd}}$ Yarmouk University, Irbid 211-63, Jordan

${ }^{\text {ee }}$ On leave from J. Stefan Institute, Ljubljana, Slovenia
} 
The $e e \gamma \gamma E_{T}$ event sparked considerable theoretical interest, because this signature is very rare in the SM and the event's topology is consistent with that of a decay of a pair of new heavy particles. In Run II, both the CDF [10] and D0 [11] collaborations searched for production of $\gamma \gamma+E_{T}$ events in the context of gauge-mediated supersymmetry breaking models using data corresponding to $0.20 \mathrm{fb}^{-1}$ and $1.1 \mathrm{fb}^{-1}$ of integrated luminosity, respectively. A search for anomalous production of $\gamma \gamma+e / \mu$ events with energetic central photons and leptons $\left(E_{T}^{\gamma, e}>\right.$ $25 \mathrm{GeV}$ and $p_{T}^{\mu}>25 \mathrm{GeV} / c$ ) in $0.93 \mathrm{fb}^{-1}$ of data was performed by the CDF collaboration as a part of a broader signature-based search for new physics in $l+\gamma+X(l=$ $e, \mu$ and $X=\gamma, l, E_{T}$ ) events [12]. Other signatures involving two photons were studied in CDF searches reported in Refs. [13-15]. The current model-independent analysis is improved upon previous diphoton searches both in terms of refined experimental techniques and of amount of data analyzed. It also probes a wider kinematic range compared to the CDF analyses reported in Refs. [12,15].

This paper is organized as follows. It begins with a description of the CDF II detector and the baseline diphoton sample. Then, each $\gamma \gamma+X\left(X=e / \mu, \tau\right.$, and $\left.E_{T}\right)$ subsample is discussed in separate sections where we describe the definition of the subsamples, the calculation of the SM predictions, and the comparison of the data and the predictions. The details of several techniques are postponed to appendices.

\section{DETECTOR OVERVIEW}

The CDF II detector is a cylindrically symmetric apparatus designed to study $p \bar{p}$ collisions at the Fermilab Tevatron. The detector has been described in detail elsewhere [16]; only the detector components that are relevant to this analysis are briefly discussed below. The magnetic spectrometer consists of tracking devices inside a 3-m diameter, 5-m long superconducting solenoid magnet which provides an axial magnetic field of $1.4 \mathrm{~T}$. A set of silicon microstrip detectors [17-19] and a 3.1-m long drift chamber (COT) [20] with 96 layers of sense wires measure momenta and trajectories (tracks) of charged particles in the pseudorapidity regions of $|\eta|<2$ and $|\eta|<1$ [3], respectively. Surrounding the magnet coil is the projective-towergeometry sampling calorimeter, which is used to identify and measure the energy and position of photons, electrons, jets, and $E_{T}$. The calorimeter consists of lead-scintillator electromagnetic and iron-scintillator hadron compartments and it is divided into a central barrel $(|\eta|<1.1)$ and a pair of "end plugs" that cover the region $1.1<|\eta|<3$.6. The central calorimeter is composed of towers with a segmentation of $\Delta \eta \times \Delta \phi \simeq 0.1 \times 15^{\circ}$. The energy resolution of the central electromagnetic calorimeter for electrons is $\sigma\left(E_{T}\right) / E_{T}=13.5 \% / \sqrt{E_{T}(\mathrm{GeV})} \oplus 1.5 \%$ [21], while the energy resolution of the central hadron calorimeter for charged pions that do not interact in the electromagnetic section is $\sigma\left(E_{T}\right) / E_{T}=50 \% / \sqrt{E_{T}(\mathrm{GeV})} \oplus 3 \%$ [22]. In the plug calorimeter, the segmentation varies from $\Delta \eta \times$ $\Delta \phi \simeq 0.1 \times 7.5^{\circ}$ for $1.1<|\eta|<1.8$ to $\Delta \eta \times \Delta \phi \simeq$ $0.6 \times 15^{\circ}$ for $|\eta|=3.6$. The corresponding plug electromagnetic and hadron calorimeter energy resolutions are $\sigma(E) / E=14.4 \% / \sqrt{E(\mathrm{GeV})} \oplus 0.7 \%$ and $\sigma(E) / E=$ $74 \% / \sqrt{E(\mathrm{GeV})} \oplus 4 \%$, respectively [23]. The additional system in the central region is used for identification and precise position measurement of photons and electrons. Multiwire proportional chambers with cathode-strip readout (the CES system) are located at the depth of six radiation lengths (near shower maximum) in the central electromagnetic calorimeter. Cathode strips and anode wires, with a channel spacing between $1.5 \mathrm{~cm}$ and $2 \mathrm{~cm}$, running along the azimuthal (strips) and the beam line (wires) direction give location and two-dimensional profiles of the electromagnetic showers. The position resolution of the CES is $2 \mathrm{~mm}$ for a $50 \mathrm{GeV}$ photon. The electromagnetic compartments of the calorimeter are also used to measure the arrival time of particles depositing energy in each tower [24]. Muons from collisions as well as cosmic rays are identified using systems which are located outside the calorimeters: the central muon detector (CMU) and the central muon upgrade detector (CMP) in the pseudorapidity region of $|\eta|<0.6$, and the central muon extension (CMX) for the pseudorapidity region of $0.6<|\eta|<1.0$ [25]. The CMU system uses four layers of planar drift chambers and detects muons with $p_{T}>1.4 \mathrm{GeV} / c$. The CMP system, located behind a $0.6 \mathrm{~m}$ thick steel absorber outside the magnetic return yoke, consists of an additional four layers of planar drift chambers and detects muons with $p_{T}>2.2 \mathrm{GeV} / c$. The CMX detects muons with $p_{T}>1.4 \mathrm{GeV} / c$ using four to eight layers of drift chambers, depending on the polar angle. A system of Cherenkov luminosity counters (CLC) [26], located around the beam pipe and inside the plug calorimeters, is used to measure a number of inelastic $p \bar{p}$ collisions per bunch crossing, and thereby the luminosity.

The online event selection at CDF is done by a threelevel trigger [27] system with each level providing a rate reduction sufficient to allow for processing at the next level with minimal deadtime. The Level-1 trigger uses custom designed hardware to find physics objects based on a subset of the detector information. The Level-2 trigger consists of custom hardware to do a limited event reconstruction which can be processed in programmable processors. The Level-3 trigger uses the full detector information and consists of a farm of computers that reconstructs the data and applies selection criteria similar to the offline requirements.

\section{DATA SELECTION AND EVENT RECONSTRUCTION}

The search for anomalous production of $\gamma \gamma+E_{T}$ and $\gamma \gamma+\tau$ events is performed with data corresponding to 
$2.0 \pm 0.1 \mathrm{fb}^{-1}$ of luminosity integrated from the beginning of Run II. The search for anomalous $\gamma \gamma+e / \mu$ events utilizes a smaller data set of $1.1 \pm 0.1 \mathrm{fb}^{-1}$ of integrated luminosity. The online (trigger) requirements and offline selection criteria that are common to all three final states are discussed below. Additional requirements of each analysis are explained separately.

The inclusive $\gamma \gamma$ events are selected online by a threelevel trigger that requires two isolated electromagnetic (EM) clusters with $E_{T}^{\gamma}>12 \mathrm{GeV}$ (diphoton-12 trigger) or two electromagnetic clusters with $E_{T}^{\gamma}>18 \mathrm{GeV}$ and no isolation requirement (diphoton-18 trigger). A detailed description of diphoton triggers can be found in Appendix A 1. The triggered $\gamma \gamma$ candidate events are then subjected to the offline selection. Each event is required to have two central EM clusters (photon candidates) inside a well-instrumented region of the calorimeter (approximately $0.05<|\eta|<1.05$ ) with $E_{T}>13 \mathrm{GeV}$. For each photon candidate, the transverse shower profile in the CES and the amount of energy leaked into the hadron calorimeter must be consistent with those of a single electromagnetic shower. We distinguish photons from electrons by making sure that no high- $p_{T}$ charged track points to the EM cluster. Both photon candidates are also required to be isolated energy clusters in the calorimeter in order to suppress background due to jets. More details of the standard photon identification criteria can be found in Appendix A 2. To reduce contamination due to cosmic-ray, beam-related, and other noncollision backgrounds, the event must contain a well-reconstructed vertex, formed by tracks, with $|z|<60 \mathrm{~cm}$. If multiple vertices are reconstructed, the vertex with the largest $\sum p_{T}$ of the associated tracks is selected. Unless noted otherwise, the transverse energy of all calorimeter objects is calculated with respect to this primary vertex. Finally, the arrival time of both photon candidates, corrected for average path length, has to be consistent with the $p \bar{p}$ collision time. It should be pointed out that due to the photon timing requirements, we are only sensitive to new physics processes where photons are produced in decays of new particles with small lifetime $(<1 \mathrm{~ns})$.

Inclusive $\gamma \gamma$ events satisfying the above criteria form the baseline $\gamma \gamma$ signal sample used in all three analyses. This sample consists of real $\gamma \gamma$ events (approximately $30 \%)$, jet- $\gamma(45 \%)$, and jet-jet (25\%) [10] events where one or both jets are misidentified as a photon. (An object misidentified as a photon is referred to as a "fake" photon.) The $\gamma \gamma+e / \mu, \gamma \gamma+\tau$, and $\gamma \gamma+E_{T}$ candidate events are then selected from the base signal sample by requiring additional objects of interest or significant $E_{T}$. We also select a control sample of $\gamma \gamma$ events by applying less stringent photon identification requirements as discussed in Appendix A 2. To avoid an overlap with the signal sample, at least one photon candidate from the control sample must fail the standard photon cuts. The control sample is ideal for testing our analysis techniques because it has a similar event topology, but is dominated by background events (the fraction of real $\gamma \gamma$ events in it is approximately $5 \%$ ).

Our baseline signal and control $\gamma \gamma$ samples consist of 31116 and 42708 events, respectively, in data corresponding to $2.0 \mathrm{fb}^{-1}$ of integrated luminosity.

\section{SEARCHES FOR ANOMALOUS PRODUCTION OF $\gamma \gamma+X$ EVENTS}

In this section, we describe in detail three separate searches for anomalous production of $\gamma \gamma+e / \mu, \gamma \gamma+$ $\tau$, and $\gamma \gamma+E_{T}$ events. All analyses use the same baseline $\gamma \gamma$ samples and utilize the same definitions of the additional objects and kinematic variables: electrons, muons, $\tau$ leptons, jets, soft unclustered energy, $E_{T}$, and $H_{T}$. The $H_{T}$ is defined as a scalar sum of $E_{T}$ and $E_{T}$ of all identified photons, leptons, and jets. The detailed descriptions of these objects can be found in Appendices A 3-A 8.

\section{A. The $\gamma \gamma+e / \mu$ final state}

We search for anomalous production of events containing two photons and at least one additional electron or muon in data corresponding to $1.1 \pm 0.1 \mathrm{fb}^{-1}$ of integrated luminosity. The events of interest are derived from the $\gamma \gamma$ baseline sample described in Sec. III. The electron identification criteria are similar to those for the photon except that an electron candidate must have an energetic track pointing to the EM cluster. The momentum, $p$, of this track has to be consistent with the energy deposited in the EM calorimeter. The electron identification requirements are described in detail in Appendix A 3.

A well-reconstructed COT track is identified as a muon candidate if it is matched to hit segments (stubs) in the central muon detectors, and its energy deposition pattern in the EM and hadron (HAD) calorimeters is consistent with that left by a minimum ionizing particle. Details on the muon identification requirements can be found in Appendix A 4.

The selected $\gamma \gamma e$ and $\gamma \gamma \mu$ events must have at least one electron or muon candidate with $E_{T}^{e}>20 \mathrm{GeV}$ and $p_{T}^{\mu}>$ $20 \mathrm{GeV} / c$, respectively. We compare the observed number of events and kinematic distributions in the data with those from our SM background predictions. Backgrounds for the $\gamma \gamma e$ and $\gamma \gamma \mu$ signatures of new physics include the following:

(1) The SM production of $Z \rightarrow l^{+} l^{-}$and $W \rightarrow l \nu$ in association with two photons $(Z \gamma \gamma, W \gamma \gamma)$, where photons are radiated from either the initial-state quarks, charged electroweak boson $(W)$, or the final-state leptons.

(2) Backgrounds due to misidentified particles (fake photons or leptons)

(i) electrons misidentified as photons (e.g., $Z \gamma$ events), 
(ii) jets misidentified as photons (e.g, $W \gamma+$ jet or $Z \gamma+$ jet events)

(iii) jets misidentified as leptons (mostly $\gamma \gamma$ candidate events with an additional jet).

We describe below how these background contributions are estimated.

The SM $Z \gamma \gamma$ and $W \gamma \gamma$ contributions are estimated from Monte Carlo (MC) simulation. The MC samples are generated using the leading-order (LO) matrix-element generator MADGRAPH [28]. The output of MADGRAPH is fed into PYTHIA [29] to carry out parton fragmentation, simulation of the underlying event and additional $p \bar{p}$ interactions in the same bunch crossing, as well as initial- and final-state radiation. The output of PYTHIA is then processed through the GEANT-based detector simulation [30] followed by the same reconstruction program as that for the data. To account for an imperfect modeling of the CDF II detector, the MC predictions are corrected for small differences $(1 \%-10 \%)$ in photon and lepton identification and trigger efficiencies between data and MC. In addition, the LO cross sections predicted by MADGRAPH are scaled to the next-to-leading-order (NLO) cross sections according to the $K$ factors in Ref. [31]. These $K$ factors are functions of the dilepton mass and $E_{T}$ of the highest- $E_{T}$ photon, and their values range from 1.36 to 1.62 with an average of $\sim 1.4$ for the kinematic range of $Z \gamma \gamma$ and $W \gamma \gamma$ production. The uncertainty for this background prediction includes statistical uncertainty due to the finite size of MC samples, $6 \%$ systematic uncertainty on the measured integrated luminosity, and $7 \%$ systematic uncertainty on the $Z \gamma \gamma$ and $W \gamma \gamma$ cross sections due to uncertainties in the factorization and renormalization scales and parton distribution functions (PDF) [32].

The rest of the background contains at least one misidentified object. Fake photons can arise from the hard bremsstrahlung of electrons in the detector material, inefficient electron-track reconstruction, or decays of $\pi^{0}, \eta^{0}$, or $K_{s}^{0}$ in jets. Although these background sources yield real photons in the final state, they are referred to as fake in this analysis, to be distinguished from the photons possibly produced by new exotic particles. The number of $\gamma \gamma+l$ $(l=e, \mu)$ events where at least one of the photons is faked by an electron is estimated from the $l \gamma+e$ data (collected with the diphoton triggers). We obtain the prediction by applying an $e \rightarrow \gamma$ misidentification probability as a function of the $E_{T}$ of electron (about 2.7\% and 1.5\% for electrons with $E_{T}=20 \mathrm{GeV}$ and $40 \mathrm{GeV}$, respectively) to the selected $l \gamma+e$ events. More details about this misidentification probability and its uncertainty are included in Appendix B 1. To estimate the number of $\gamma \gamma+l$ events where at least one of the photons is a misidentified jet, we select the $l \gamma+$ jet data collected with inclusive lepton triggers and multiply them by a jet $\rightarrow \gamma$ misidentification probability as a function of the jet's $E_{T}$ (about $0.2 \%$ and
$0.04 \%$ for jets with $E_{T}=13 \mathrm{GeV}$ and $>50 \mathrm{GeV}$, respectively). The description of the jet $\rightarrow \gamma$ misidentification probability and its associated uncertainty can be found in Appendix B 2. Also note that both $l \gamma+e$ and $l \gamma+$ jet samples may contain events with fake leptons.

The last source of background is events with two real photons and a fake lepton from the direct diphoton production with additional jets. The number of "diphoton + fake lepton" events is obtained by applying $E_{T}$-dependent misidentification probabilities from Ref. [33] to the events with two photon candidates and an object which may fake a lepton. These objects are jets for electrons and isolated tracks for muons. The probability for a jet (isolated track) with $E_{T}\left(p_{T}\right)=50 \mathrm{GeV}$ to fake a central electron (muon) is $\sim 0.01 \%(\sim 1 \%)$. Details of the misidentification probabilities and their uncertainties are discussed in Appendix B 3. According to earlier studies [10], only $29 \% \pm 4 \%$ of observed diphotons are real diphoton events. In order to avoid duplication with the fake photon contribution estimated above, the number of "diphoton + fake lepton" events is multiplied by the real diphoton fraction $(29 \% \pm 4 \%)$, which gives the number of "real $\gamma \gamma+$ fake lepton" events.

The fake photon signature can also be produced as a result of the bremsstrahlung of cosmic muons as they pass through the calorimeters. However, the probability for a real photon event to overlap with such a cosmic event is found to be very small: $1.5 \times 10^{-8}$ (see Ref. [10]). Therefore, the cosmic backgrounds are negligible in the $\gamma \gamma e$ and $\gamma \gamma \mu$ searches.

Table I lists the expected and observed numbers of $\gamma \gamma e$ and $\gamma \gamma \mu$ events for $E_{T}^{\gamma}>13 \mathrm{GeV}$. At this stage of event selection, we observe three $\gamma \gamma e$ events and zero $\gamma \gamma \mu$ events. The leading background in the $\gamma \gamma e$ channel is due to events where at least one of the photons is a misidentified electron. The leading background in the $\gamma \gamma \mu$ channel is the electroweak production of $Z \gamma \gamma$ events. Figures 1 and 2 show several important kinematic distributions, including invariant mass, electron and photon $E_{T}$, $E_{T}$, jet multiplicity, and $H_{T}$ from data and the predicted

TABLE I. Summary of the predicted and observed numbers of $\gamma \gamma e$ and $\gamma \gamma \mu$ events before applying silicon-track rejection. The systematic uncertainty includes uncertainty due to MC statistics, uncertainties in the data luminosity, predicted cross sections, and the misidentification probabilities.

\begin{tabular}{lcc}
\hline \hline Source & Electron & Muon \\
\hline$Z \gamma \gamma$ & $0.90 \pm 0.09$ & $0.55 \pm 0.05$ \\
$W \gamma \gamma$ & $0.17 \pm 0.02$ & $0.09 \pm 0.01$ \\
$l \gamma+e \rightarrow \gamma$ & $5.14 \pm 0.68$ & $0.02 \pm 0.02$ \\
$l \gamma+$ jet $\rightarrow \gamma$ & $0.48 \pm 0.31$ & $0.13 \pm 0.09$ \\
Fake $l+\gamma \gamma$ & $0.13 \pm 0.05$ & $0.004 \pm 0.004$ \\
Total & $6.82 \pm 0.75$ & $0.79 \pm 0.11$ \\
Data & 3 & 0 \\
\hline \hline
\end{tabular}



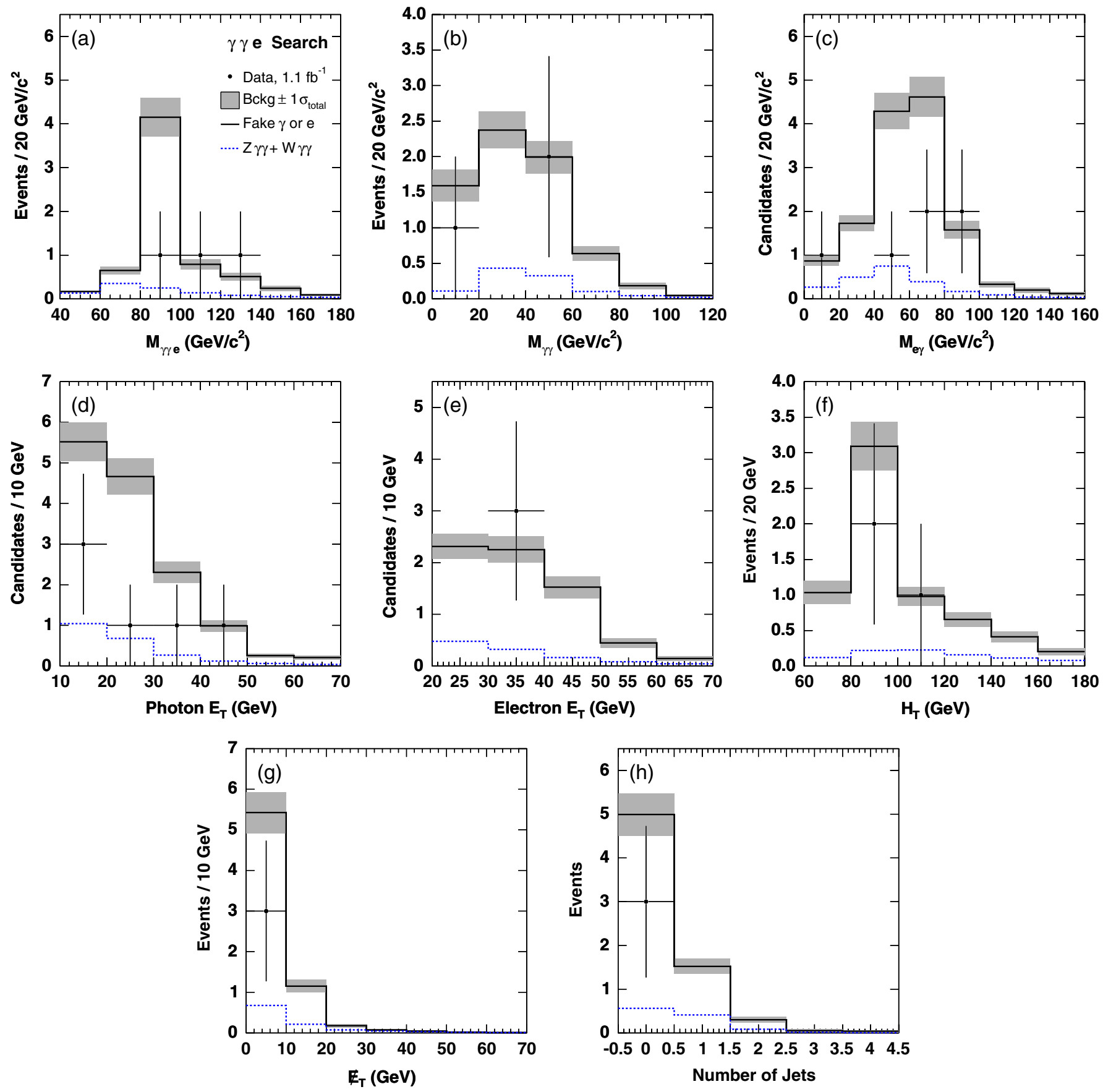

FIG. 1 (color online). Kinematic distributions of the $\gamma \gamma$ e events from the SM (dashed line) and total (solid line) background predictions as well as the three events observed in the data (marker). The total background includes SM and fake contributions. The gray boxes indicate the uncertainty in background determination. Each photon is required to have an $E_{T}>13 \mathrm{GeV}$. Distributions from the top left to the bottom right are (a) three-body invariant mass; (b) invariant mass of two photons; (c) invariant mass of each electronphoton pair; (d) $E_{T}$ of each photon; (e) $E_{T}$ of the electron; (f) $H_{T}$, scalar sum of $E_{T}$ and $E_{T}$ of all identified photons, electrons, and jets; (g) $E_{T}$; and (h) number of jets with $E_{T}>15 \mathrm{GeV}$.

backgrounds before applying the final selection, the silicon-track rejection (described next).

The dominant source of background in the $\gamma \gamma e$ search are $\gamma+e e$ events where one of the electrons is misreconstructed as a photon. An electron may lose its track and be reconstructed as a photon because of catastrophic bremsstrahlung in the detector material in front of the COT. However, such an electron often leaves a few hits in the silicon detector and can be partially recovered by a special tracking algorithm (see Appendix A 2 and Ref. [34] for more details). We further compare the data and background prediction after removing events where at least one 

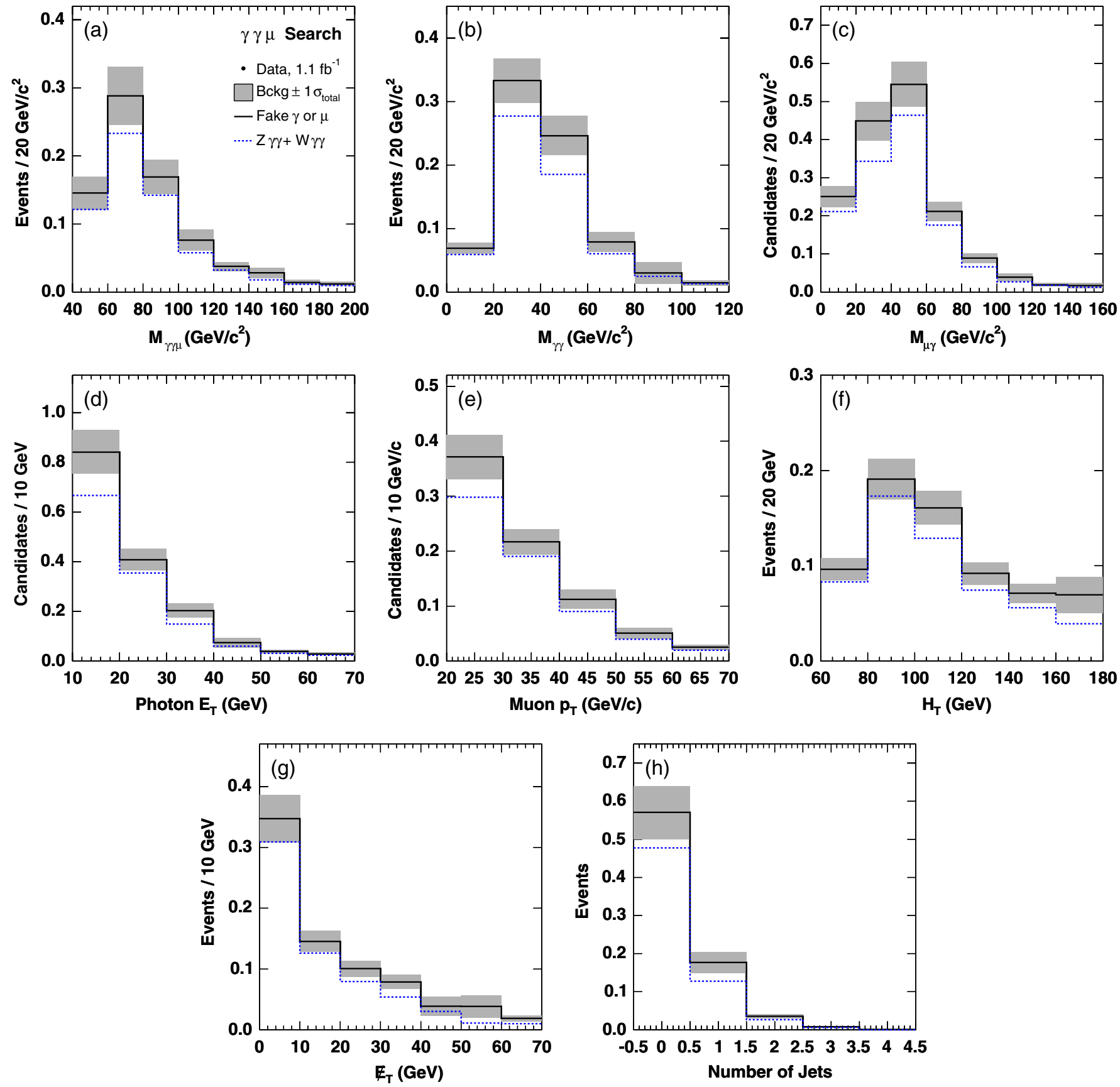

FIG. 2 (color online). Kinematic distributions of the $\gamma \gamma$ e events from the SM (dashed line) and total (solid line) background predictions. The total background includes SM and fake contributions. The gray boxes indicate the uncertainty in background determination. We observe zero events in the data. Each photon is required to have an $E_{T}>13 \mathrm{GeV}$. Distributions from the top left to the bottom right are (a) three-body invariant mass; (b) invariant mass of two photons; (c) invariant mass of each muon-photon pair; (d) $E_{T}$ of each photon; (e) $p_{T}$ of the muon; (f) $H_{T}$, scalar sum of $E_{T}$ and $E_{T}$ of all identified photons, muons, and jets; (g) $E_{T}$; and (h) number of jets with $E_{T}>15 \mathrm{GeV}$.

of the photons is matched to this type of electron track (silicon-track rejection). The silicon-track rejection suppresses $\sim 80 \%$ of fake photons from the electron bremsstrahlung for $E_{T}^{\gamma}>45 \mathrm{GeV}$ (see Fig. 12 from Appendix B 3 ) while it has only $\sim 1 \%$ inefficiency for real photons. Once this procedure is applied, the observed number of $\gamma \gamma e$ events is reduced to one. The final background predictions after the silicon-track rejection can be found in Table II.

The robustness of our background estimation technique is validated in the following three ways. First, we use an independent method to measure the misidentification 
TABLE II. Summary of the predicted and observed numbers of $\gamma \gamma e$ and $\gamma \gamma \mu$ events after applying silicon-track rejection. The systematic uncertainty includes uncertainty due to MC statistics, uncertainties in the data luminosity, predicted cross sections, and the misidentification probabilities.

\begin{tabular}{lcc}
\hline \hline Source & Electron & Muon \\
\hline$Z \gamma \gamma$ & $0.82 \pm 0.08$ & $0.50 \pm 0.05$ \\
$W \gamma \gamma$ & $0.15 \pm 0.02$ & $0.08 \pm 0.01$ \\
$l \gamma+e \rightarrow \gamma$ & $2.26 \pm 0.46$ & $0.004 \pm 0.004$ \\
$l \gamma+$ jet $\rightarrow \gamma$ & $0.44 \pm 0.26$ & $0.12 \pm 0.08$ \\
Fake $l+\gamma \gamma$ & $0.12 \pm 0.05$ & $0.004 \pm 0.004$ \\
Total & $3.79 \pm 0.54$ & $0.71 \pm 0.10$ \\
Data & 1 & 0 \\
\hline \hline
\end{tabular}

probabilities. Instead of counting electrons that satisfy standard central-electron criteria, we use the number of electrons that satisfy photonlike electron criteria in the $e \rightarrow \gamma$ fake rate denominator (see Appendix B 1). The difference in the prediction of fake photons from eer events is $\sim 4 \%-11 \%$. Second, we cross-check if the eer data (to which the $e \rightarrow \gamma$ fake rate is applied) contain a significant number of fake electrons. We fit data with a combined likelihood of multiple electron identification variables, where the signal shapes are obtained from electrons in $Z$ decays and the background shapes are obtained from the sample enriched with fake electrons. The purity of electrons is estimated to be $97 \pm 2 \%$. In addition, we compare the yields of eer and $\mu \mu \gamma$ events in data and those predicted by $Z \gamma$ MADGRAPH MC. We divide the ratio of data to $\mathrm{MC}$ yields in the muon channel by the same ratio in the electron channel. If the ee $\gamma$ events contain a significant amount of fake electrons, the double ratio will be inconsistent with unity. The double ratio is found to be $1.10 \pm 0.15$. Third, we examine the background estimate in larger samples, where either one photon and one electron $(e \gamma)$ or one photon, one electron, and one jet $(e \gamma j)$ events are required. The numbers of $e \gamma$ and $e \gamma j$ events in data are consistent with those from the background predictions within 1 standard deviation.

To summarize, we do not observe any evidence for anomalous production of $\gamma \gamma e$ and $\gamma \gamma \mu$ events.

\section{B. The $\gamma \gamma+\tau$ final state}

We search for events with two photons and a hadronically decaying $\tau$ lepton in data corresponding to $2.0 \mathrm{fb}^{-1}$ of integrated luminosity. These events are a subset of the baseline diphoton sample (see Sec. III) with at least one $\tau$ lepton candidate identified using the tight requirements and passing $E_{T}>15 \mathrm{GeV}$ (see Appendix A 5). We select 34 $\gamma \gamma+\tau$ candidate events.

We consider two sources of backgrounds: the SM production of $W \rightarrow \tau \nu$ or $Z \rightarrow \tau \tau$ with photons and $\gamma \gamma$ events with jets misidentified as $\tau$ leptons. Other backgrounds are negligible.
The electroweak backgrounds are estimated from $W \gamma$ and $Z \gamma$ MADGRAPH [28] MC simulation. The LO order predictions are multiplied by the appropriate next-to-leading-order $K$ factors described in Sec. IVA and Ref. [31]. We find that these electroweak events with real leptons are dominated by events with at least one real photon, so we do not consider the case where both photons are misidentified jets. The simulation predicts the background from the cases of two real photons or one real with one fake photon to be $2.2 \pm 0.8$ events, where the uncertainty comes from MC statistics.

The dominant background in this search is from events with two reconstructed photons (which may be real or fake), and jets, where one of the jets is misidentified as a $\tau$ lepton. To estimate this background, we select events with two photons and a jet identified as a "loose" $\tau$ lepton candidate (see Appendix A 5) and apply the jet $\rightarrow \tau$ misidentification probability (see Appendix B 4). Since the misidentification probability is different for jets originated by quarks or by gluons, and the ratio of quark jets to gluon jets here may be different from the one in the sample used to derive the jet $\rightarrow \tau$ misidentification probability, we investigate a correction for the different types of jets in our sample. The probability for a quark jet to fake a $\tau$ lepton is 3 times larger than the probability for a gluon jet. The process becomes more complex because a photon candidate may also be a misidentified jet, and the probability for a quark jet to fake a photon is 10 times larger than for a gluon jet. We use PYTHIA MC samples of diphotons, inclusive single photons and dijets to investigate the quark and gluon content of our data sample. Previous studies [10] have determined that the baseline diphoton sample has approximately $30 \%$ real diphoton events, $45 \%$ events with a real photon and a jet misidentifed as a photon, and $25 \%$ events with two jets misidentified as photons. The simulations indicate that the quark-to-gluon ratio is significantly higher in the case of one real photon and one fake photon (80\% quarks) than either of the other cases (approximately $30 \%$ in dijet events and $40 \%$ in events with two real photons) and needs to be corrected for. We account for this effect by using two methods. In the first method, we simply apply the jet $\rightarrow \tau$ misidentification rate and then make a correction for the difference in the average quark-to-gluon ratio in the sample. In the second method, we allow for the possibility that quark jets will preferentially become misidentified photons, leaving the remaining jets to become misidentified taus. This method yields our reported central result, and the variation between the methods indicates a $13 \%$ systematic uncertainty which is added to the $20 \%$ systematic uncertainty in the misidentification probability.

The misidentified $\tau$ background is $44 \pm 10$ events and the total background estimate is $46 \pm 10$ events, consistent with the 34 observed $\gamma \gamma+\tau$ candidate events. We perform three checks of the methodology by predicting the size of 


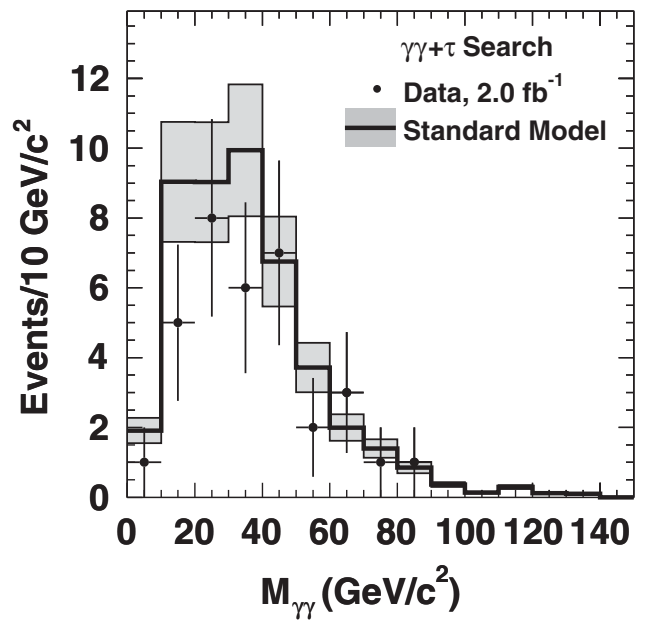

FIG. 3. The mass of the two photons in $\gamma \gamma+\tau$ candidate events (marker) and the SM backgrounds (histogram). The gray boxes indicate the uncertainty in background determination.

the $\gamma \gamma+\tau$ sample where the two photons are selected with the relaxed criteria ( $\gamma \gamma$ control sample described in Sec. III) or with one of the photons in the forward region $(1.1<|\eta|<2.0)$. The predictions for all control samples are consistent with the observations. Figures 3 and 4 show several important kinematic distributions for the selected $\gamma \gamma+\tau$ candidate events and the predicted SM background. These distributions include the diphoton invariant mass, $E_{T}$ of a $\tau$ lepton candidate, $E_{T}$, jet multiplicity, and $H_{T}$. No excess is found above the SM background.
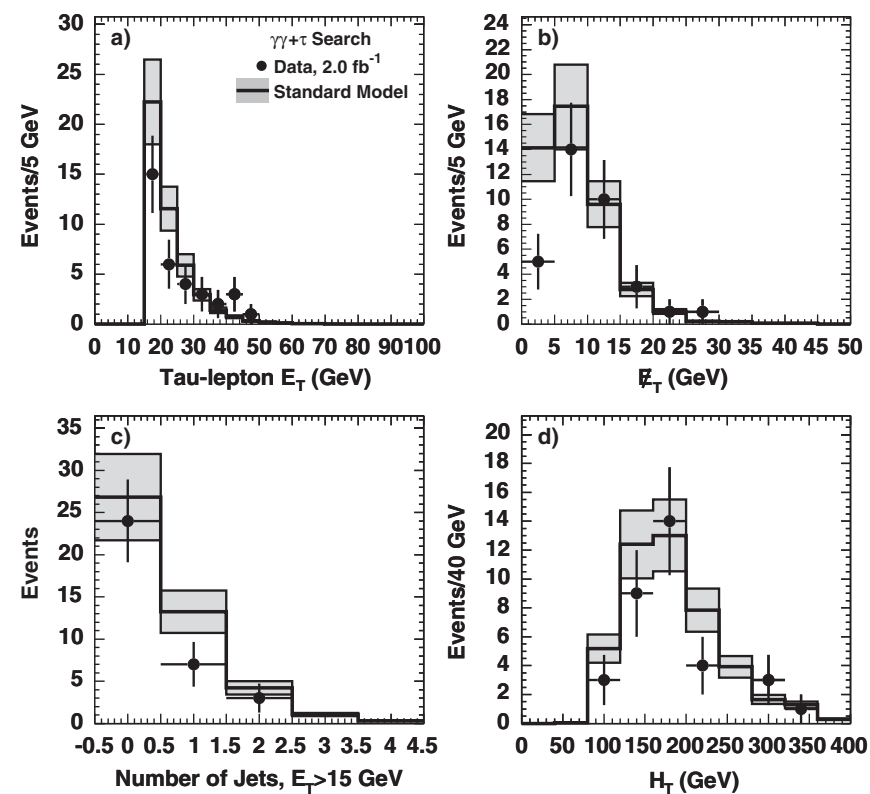

FIG. 4. The kinematic distributions in $\gamma \gamma+\tau$ candidate events (marker) and the SM backgrounds (histogram): (a) $E_{T}$ of $\tau$ lepton candidate; (b) $E_{T}$; (c) number of jets with $E_{T}>$ $15 \mathrm{GeV}$; (d) $H_{T}$, scalar sum of the transverse energies of photons, $\tau$ lepton candidate, jets, and $E_{T}$. The gray boxes indicate the uncertainty in background determination.
In summary, we do not observe any evidence for the anomalous production of $\gamma \gamma+\tau$ events.

\section{The $\gamma \gamma+E_{T}$ final state}

We search for the anomalous production of two photons and large missing transverse energy $\left(E_{T}\right)$ in data corresponding to $2.0 \mathrm{fb}^{-1}$ of integrated luminosity. The subsample of $\gamma \gamma+E_{T}$ events is derived from the baseline diphoton sample described previously in Sec. III and in Appendix A 2. The missing transverse energy is defined as an energy imbalance in the calorimeter (see detailed description of $E_{T}$ in Appendix A 7) and it is an experimental signature of neutrinos or new particles that do not interact electromagnetically or strongly with the detector material. The $E_{T}$, however, can be mimicked by a simple energy misreconstruction in SM events. Fluctuations in jet energy measurements are the most common source of such fake $E_{T}$. Figure 5 shows the $E_{T}$ distribution in the $\gamma \gamma$ baseline sample. This figure illustrates that events with fake $E_{T}$ are not only the dominant background in the region up to $\mathbb{E}_{T} \sim$ $40 \mathrm{GeV}$, but they also have a significant contribution even to the tail of $E_{T}$ distribution. A better separation between events with real and fake $E_{T}$ can be achieved if a significance of the measured $E_{T}$ is considered rather than its absolute value. The $E_{T}$-significance is a dimensionless quantity based on the energy resolution of jets and soft unclustered particles. It also takes into account the event topology as shown in Appendix C. As it is demonstrated in Fig. 6, the $E_{T}$-significance distributions have very different shapes in events with fake and real $E_{T}$ : exponentially falling (solid line) and almost flat shapes, respectively. Thus, the $E_{T}$-significance is an efficient tool in separating

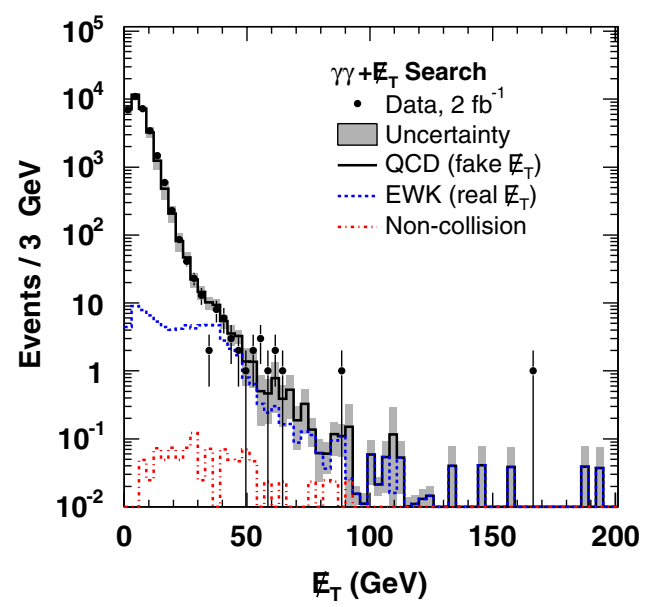

FIG. 5 (color online). The $E_{T}$ distribution in all $\gamma \gamma$ candidate events from the baseline sample. The data (marker) is compared with the total background prediction (solid line with the gray band representing the total uncertainty). The total background prediction is a sum (shown by the stacked histograms) of the QCD, electroweak (dashed line), and noncollision (dasheddotted line) backgrounds. 


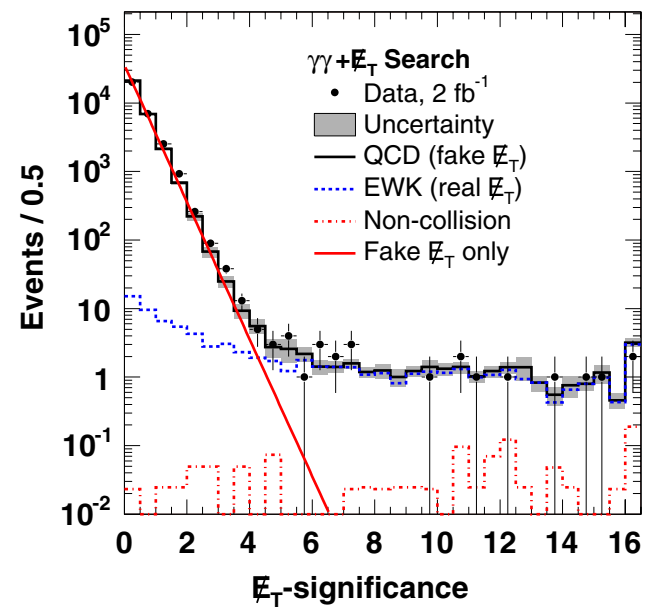

FIG. 6 (color online). The $E_{T}$-significance distribution in all $\gamma \gamma$ candidate events from the baseline sample. The data (marker) is compared with the total background prediction (solid line with the gray band representing the total uncertainty). The total background prediction is a sum (shown by the stacked histograms) of the QCD, electroweak (dashed line), and noncollision (dashed-dotted line) backgrounds. The straight solid line represents the expected $E_{T}$-significance distribution if all $\gamma \gamma$ candidate events were to have fake $E_{T}$ due to the measurement fluctuations in the calorimeter (see Appendix $\mathrm{C}$ for more details).

such events. We study $\gamma \gamma+E_{T}$ events which pass three a priori $E_{T}$-significance requirements: $E_{T}$-significance $>$ 3,4 , and 5 . This choice of cut values has a straightforward motivation. If the $\gamma \gamma$ sample were only composed of events with fake $E_{T}$ due to energy misreconstruction in the calorimeter, then we would select $0.1 \%, 0.01 \%$, and $0.001 \%$ of the total number of events by requiring $E_{T}$-significance $>3,4$, and 5, respectively. On the other hand, studies with MC $W \gamma \rightarrow e \nu+\gamma$ sample indicate that the $E_{T}$-significance $>3 \quad\left(E_{T}\right.$-significance $\left.>5\right)$ cut is $\sim 100 \%(\sim 90 \%)$ efficient for events with real $E_{T}>$ $35 \mathrm{GeV}$ (see Fig. 7).

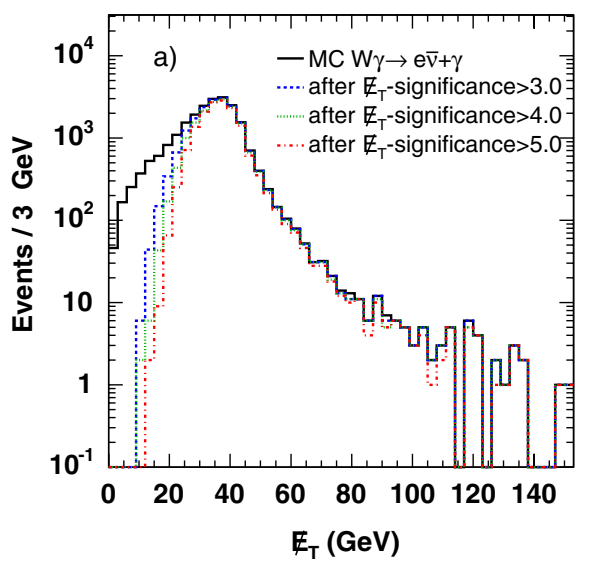

We consider three major sources of background for the $\gamma \gamma+E_{T}$ signature: QCD $(\gamma \gamma, j \gamma$, and $j j$ where $j=$ jet $\rightarrow$ $\gamma_{\text {fake }}$ ) events with large fake $E_{T}$ due to energy loss or mismeasurement in the calorimeter, electroweak (EWK) processes with real $E_{T}$ from neutrinos, and noncollision events with fake photons and $E_{T}$. Each of these sources is discussed below in the order of their importance. All of the background estimation techniques are tested on a control sample of loose diphoton events described in Sec. III and in Appendix A 2.

Significant losses or fluctuations in energy measurements in the inclusive $\gamma \gamma$ sample, which can lead to considerable values of fake $E_{T}$, happen only in a small fraction of events. However, the large production cross sections of QCD processes make them one of the largest backgrounds. We distinguish three types of QCD backgrounds: events with energy mismeasurement due to calorimeter energy resolution effects (QCD type-1); $\gamma \gamma$ candidate events with a wrong choice of the primary interaction vertex (QCD type-2); and $\gamma \gamma \gamma$ events where one of the photon candidates is lost in the calorimeter cracks (QCD type-3).

The QCD type-1 background estimate is based on a $E_{T}$-resolution model (METMODEL) described in Appendix C. For each $\gamma \gamma$ data event, we generate ten pseudoexperiments to simulate fake $E_{T}$ and calculate its significance given the event kinematics. In each pseudoexperiment, we smear the energies of jets and soft unclustered particles using appropriate resolutions. The difference between the smeared and measured energy of the object is taken as its individual contribution to the total fake $E_{T}$. We predict the QCD background due to energy mismeasurements by counting the number of pseudoexperiments that pass our $E_{T}$-significance requirements divided by the number of pseudoexperiments per event. Kinematic distributions from these pseudoexperiments are then used as QCD background templates for data. The systematic uncertainty $\left(23 \%\right.$ for $E_{T}$-significance $>3,47 \%$ for $E_{T}$-significance $>4$, and $130 \%$ for $E_{T}$-significance $>5$ )

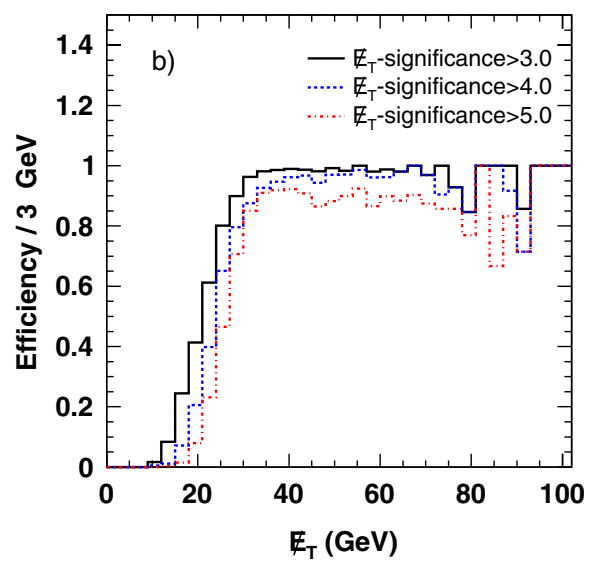

FIG. 7 (color online). (a) the $E_{T}$ distributions in $\mathrm{MC} W \gamma \rightarrow e \nu+\gamma$ events before and after the $E_{T}$-significance cuts. (b) the efficiency of the three $E_{T}$-significance cuts for these events. 
is evaluated by comparing the METMODEL expectations obtained with the default parameters to predictions obtained by varying each parameter by 1 standard deviation $( \pm 1 \sigma)$. These parameters and associated sources of systematic uncertainties are discussed in Appendix C. The statistical and systematic uncertainties are added in quadrature to obtain the total uncertainty. The predictions for the QCD type-1 background and their associated uncertainties can be found in Table III.

The background contribution due to $\gamma \gamma$ candidate events with misassigned primary vertex (QCD type-2) cannot be directly estimated by the METMODEL because the energy resolution parameterization does not include this effect. The vertex misassignment occurs when a $\gamma \gamma$ pair [35] is produced by a hard scattering interaction that overlaps with another interaction producing a vertex with the highest $\sum p_{T}$ of tracks. As a consequence of the wrong vertex choice, the $E_{T}$ of both photon candidates are incorrectly calculated, thus leading to fake $E_{T}$. Although the effect is small, it can occasionally result in a large fake $E_{T}$, for example, when two vertices are far apart and the photons are sufficiently energetic. We correct for these mismeasurements by recalculating the $E_{T}$ of photons with respect to the vertex which gives the smallest value of $E_{T}$. This procedure is verified to be valid for events with no intrinsic $E_{T}$. It is also tested in simulated $W \gamma \rightarrow e \nu \gamma$ events [36] and data $e \gamma$ events with $E_{T}>20 \mathrm{GeV}$. The selection of $e \gamma$ events is discussed in Appendix A 9. The effect is found to be small: after the procedure is applied, the number of simulated and data events with $E_{T}>20 \mathrm{GeV}$ is reduced by $1 \%$ and $2 \%$, respectively. In some fraction of events, however, the hard interaction completely fails to produce a reconstructed vertex and the vertex reassignment cannot fix fake $E_{T}$. Since the METMODEL cannot account for this contribution, we employ a method based on a combination of data and Monte Carlo simulation to obtain the predictions. For this purpose, we use PYTHIA $\gamma \gamma$ events [29] passed through the detector simulation [30]. These MC events also include additional interactions in the same bunch crossing that are modeled according to the luminosity profile in data. We select only events where the hard scattering interaction resulting in a $\gamma \gamma$ pair does not produce a reconstructed vertex, and the primary vertex is created by tracks from an overlapping additional interaction. We will refer to such events as "no vertex" $\gamma \gamma$ events. The MC sample of "no vertex" events is normalized to the number of such events in real data $(4.8 \pm 0.4 \%$ of the baseline $\gamma \gamma$ events). We then apply the standard analysis procedure to the sample and obtain the fraction of "no vertex" events in MC passing our $E_{T}$-significance cuts. The systematic uncertainties on the QCD type- 2 background contribution include the MC statistical uncertainty $(12 \%-24 \%)$, the uncertainty on the normalization factor $(10 \%)$, the uncertainty due to the jet energy scale (7\%-8\%), and the MC-data differences in the METMODEL parameterization (40\%). The predictions for the QCD type-2 background and their associated uncertainties can be found in Table III.

The $\gamma \gamma \gamma$ events are produced at a very low rate compared to that of $\gamma \gamma$ events. However a probability of losing a photon in calorimeter cracks is $\sim 10 \%$, so that the probability of losing one of the candidate photons in a triphoton event is as large as $\sim 30 \%$. These events (QCD type-3) could reconstruct as $\gamma \gamma+E_{T}$ events. To reduce this background, we reject events if the $E_{T}$ vector points along the direction (within $|\Delta \phi|<0.3$ ) of a narrow jet [38] located close to the calorimeter cracks at $\eta \sim 0$ and $|\eta| \sim 1$. 1 . The remaining contribution of the QCD type-3 events is estimated using a large inclusive PYTHIA $\gamma \gamma \mathrm{MC}$ sample. We select reconstructed triphoton events $\left(E_{T}^{\gamma 1,2}>13 \mathrm{GeV}\right.$ and $\left.E_{T}^{\gamma 3}>7 \mathrm{GeV}\right)$ in $\mathrm{MC}$ and data. The numbers of reconstructed $\gamma \gamma \gamma$ candidates give us the MC-to-data normalization factor. To obtain an estimate of the remaining QCD type-3 background, we select PYTHIA triphoton events at the generator level (before detector simulation), apply the standard analysis procedure to these events, and multiply the result by the normalization factor described above. The systematic uncertainties for this background prediction is due to the following sources: (1) MC statistical uncertainty $(24 \%-33 \%)$; (2) uncertainty on the normalization factor (19\%); (3) uncertainty due to MC-data differences in the METMODEL parameterization (10\%-44\%); (4) jet energy

TABLE III. The results of the search for anomalous production of $\gamma \gamma+E_{T}$ events. The data is compared to the background predictions for three values of the $E_{T}$-significance cut. The quoted uncertainties include the effect of limited MC statistics as well as systematic uncertainties.

\begin{tabular}{lccc}
\hline \hline & $E_{T}$-significance $>3.0$ & $E_{T}$-significance $>4.0$ & $E_{T}$-significance $>5.0$ \\
\hline EWK & $35.4 \pm 2.2$ & $29.9 \pm 2.0$ & $25.9 \pm 1.9$ \\
QCD type-1 & $28.1 \pm 6.8$ & $3.6 \pm 1.8$ & $0.6 \pm 0.8$ \\
QCD type-2 & $4.4 \pm 2.0$ & $2.5 \pm 1.0$ & $1.5 \pm 0.7$ \\
QCD type-3 & $2.9 \pm 1.0$ & $2.2 \pm 1.0$ & $1.6 \pm 1.0$ \\
Noncollision & $0.9 \pm 0.3$ & $0.8 \pm 0.3$ & $0.8 \pm 0.3$ \\
Total & $71.7 \pm 7.5$ & $39.0 \pm 3.1$ & $30.4 \pm 2.4$ \\
Data & 82 & 31 & 23 \\
\hline \hline
\end{tabular}


scale uncertainty $(10 \%-11 \%)$. The predictions for all sources of QCD backgrounds and their associated uncertainties can be found in Table III.

Electroweak processes involving $W \rightarrow l \bar{\nu}$ and $Z \rightarrow$ $\nu \bar{\nu} / \tau^{+} \tau^{-}$are the most common source of large real $E_{T}$ in $p \bar{p}$ collisions. There are three ways these processes can produce a $\gamma \gamma+E_{T}$ signature (listed in the order of importance): (1) $W \gamma$ and $Z \gamma$ events with one real and one fake photon; (2) $W \gamma \gamma$ and $Z \gamma \gamma$ events where both photons are real; (3) $W+$ jet, $Z \rightarrow \tau^{+} \tau^{-}$, and $Z+$ jet events where both photon candidates are fake photons. We estimate the EWK backgrounds by using $W / Z+\gamma$ [36] [for (1) and (2)] and inclusive $W / Z$ [39] [for (3)] Monte Carlo events passed through the detector simulation. We consider all three leptonic decay modes of $W$ and $Z$ bosons. To avoid an overlap between $W / Z+\gamma$ and $W / Z$ samples, we remove PYTHIA $W / Z$ events where reconstructed photons are matched to generated photons originating from initial/final-state radiation of quarks or leptons. The MC-based predictions for the EWK backgrounds are then multiplied by a scale factor that diminishes possible data-MC differences and cancels out many of the systematic uncertainties (e.g., trigger efficiencies, acceptance and photon ID efficiencies, $K$ factors, modeling of initial/final-state radiation in MC, uncertainties in parton distribution functions, jet energy scale uncertainty, and luminosity uncertainty). This scale factor is obtained by comparing $e \gamma+E_{T}$ events (see Appendix A 9) in data and $\mathrm{MC}$. It is defined as the ratio of numbers of data and $\mathrm{MC} e \gamma$ events satisfying all analysis requirements. The resulting EWK background predictions and the corresponding uncertainties can be found in Table III. The total uncertainties includes the MC statistical uncertainties $(3.5 \%-4.4 \%)$ and the MC-to-data normalization factor uncertainties $(5.4 \%$ $6.1 \%$ ). The last uncertainty includes statistical uncertainties from data and $\mathrm{MC} e \gamma+E_{T}$ samples and systematic uncertainties associated with the purity of the $e+\gamma$ data sample and difference between the $E_{T}$-cut efficiency (see Appendix A 9) in data and MC. From Table III, one can see that the EWK processes are the dominant source of background when $E_{T}$-significance $>4$. We find that $59 \%-$ $63 \%(30 \%-40 \%)$ of the total EWK background for the $\gamma \gamma+E_{T}$ signature comes from the electron $(\tau$ lepton)
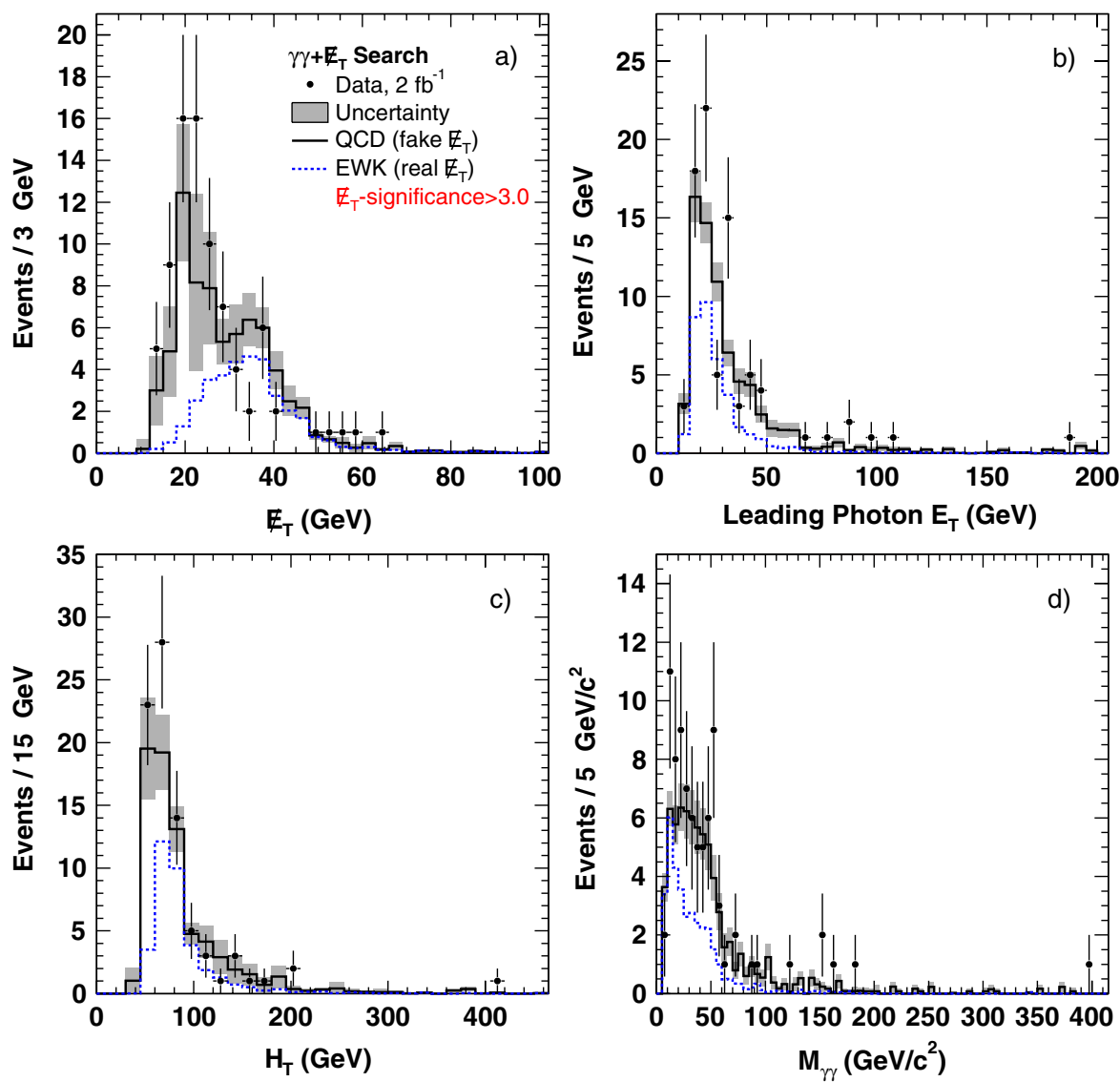

FIG. 8 (color online). The kinematic distributions for $\gamma \gamma+E_{T}$ candidate events with $E_{T}$-significance $>3$ : (a) $E_{T}$, the missing transverse energy; (b) $E_{T}$ of leading photon candidate; (c) $H_{T}$, the scalar sum of the transverse energies of photons, jets, and $E_{T}$; and (d) invariant mass, $M_{\gamma \gamma}$, of two photons. In all figures, the data (marker) is compared with the total background predictions (solid line with the gray band representing the total uncertainty). The total background prediction is a sum (shown by the stacked histograms) of the QCD and electroweak (dashed line) backgrounds. The noncollision background is too small to be visible on a plot with linear scale. 

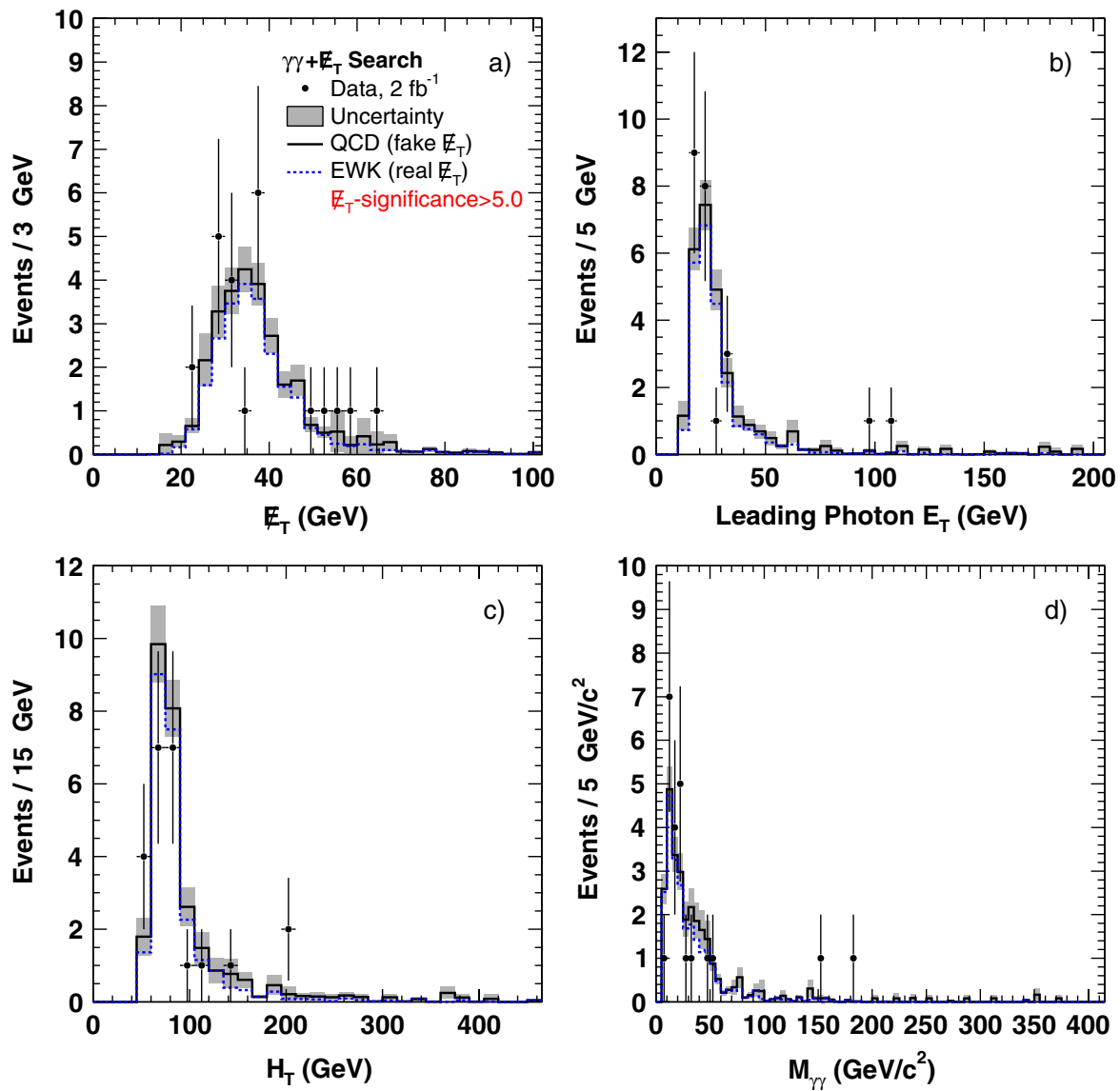

FIG. 9 (color online). The kinematic distributions for $\gamma \gamma+E_{T}$ candidate events with $E_{T}$-significance $>5$ : (a) $E_{T}$, the missing transverse energy; (b) $E_{T}$ of leading photon candidate; (c) $H_{T}$, the scalar sum of the transverse energies of photons, jets, and $E_{T}$; and (d) invariant mass, $M_{\gamma \gamma}$, of two photons. In all figures, the data (marker) is compared with the total background predictions (solid line with the gray band representing the total uncertainty). The total background prediction is a sum (shown by the stacked histograms) of the QCD and electroweak (dashed line) backgrounds. The noncollision background is too small to be visible on a plot with linear scale.

decay channels of $W$ and $Z$ bosons. Note that the $E_{T}$-significance cuts are rather efficient for events with large real $E_{T}$ : for example, $84 \%$ and $68 \%$ of $W+\gamma \rightarrow e \nu+\gamma$ events pass the $E_{T}$-significance $>3$ and $E_{T}$-significance $>$ 5 requirements, respectively.

The last remaining source of background is noncollision events where both photons and $E_{T}$ are fake. These events may either be caused by cosmic rays (CR) or beam halo (BH) muons depositing energy in the calorimeter. CR events are suppressed by requiring the EM timing of both photon candidates $\left(T_{1}\right.$ and $\left.T_{2}\right)$ to be consistent with the collision time: $\left|T_{1,2}\right|<6.7 \mathrm{~ns}$ and $\left|T_{1}-T_{2}\right|<4.1 \mathrm{~ns}$ (more details are given in Appendix A 2). BH events are removed by the topological cuts based on the distinct energy deposition pattern of $\mathrm{BH}$ muons traveling along the beam pipe. More details about $\mathrm{CR}$ and $\mathrm{BH}$ rejection cuts can be found in Appendix A 2. The number of remaining $\mathrm{BH}$ events is estimated from the number of identified $\mathrm{BH}$ candidates and known rejection power of the $\mathrm{BH}$ cuts. The background contribution due to CR events is estimated based on the number of these events in the $30 \mathrm{~ns}<T_{1,2}<$ 120 ns EM timing window and known efficiency of the cosmic rejection cuts (see Appendix A 2 and Ref. [40]). The total prediction for noncollision backgrounds can be found in Table III. The uncertainty for this estimate is dominated by the statistics in the samples of identified $\mathrm{BH}$ and $\mathrm{CR}$ events.

The results of the search are presented in Table III. The total expected SM background for three $E_{T}$-significance cuts $\left(E_{T}\right.$-significance $>3,4$, and 5$)$ is $71.7 \pm 7.5,39.0 \pm$ 3.1 , and $30.4 \pm 2.4$ events, respectively. These predictions agree well with the observed numbers of data events: 82 , 31 , and 23 . We also examine various kinematic distributions in data and SM backgrounds for $E_{T}$-significance $>3$ and $E_{T}$-significance $>5$. Figures 8 and 9 show the $E_{T}$, leading photon $E_{T}, H_{T}$, and $M_{\gamma \gamma}$ distributions for the selected $\gamma \gamma+E_{T}$ candidate events and the SM background predictions. Finally, Fig. 10 illustrates multiplicities and $E_{T}$ distributions of extra jets and electrons in selected events. We observe good agreement between data and 

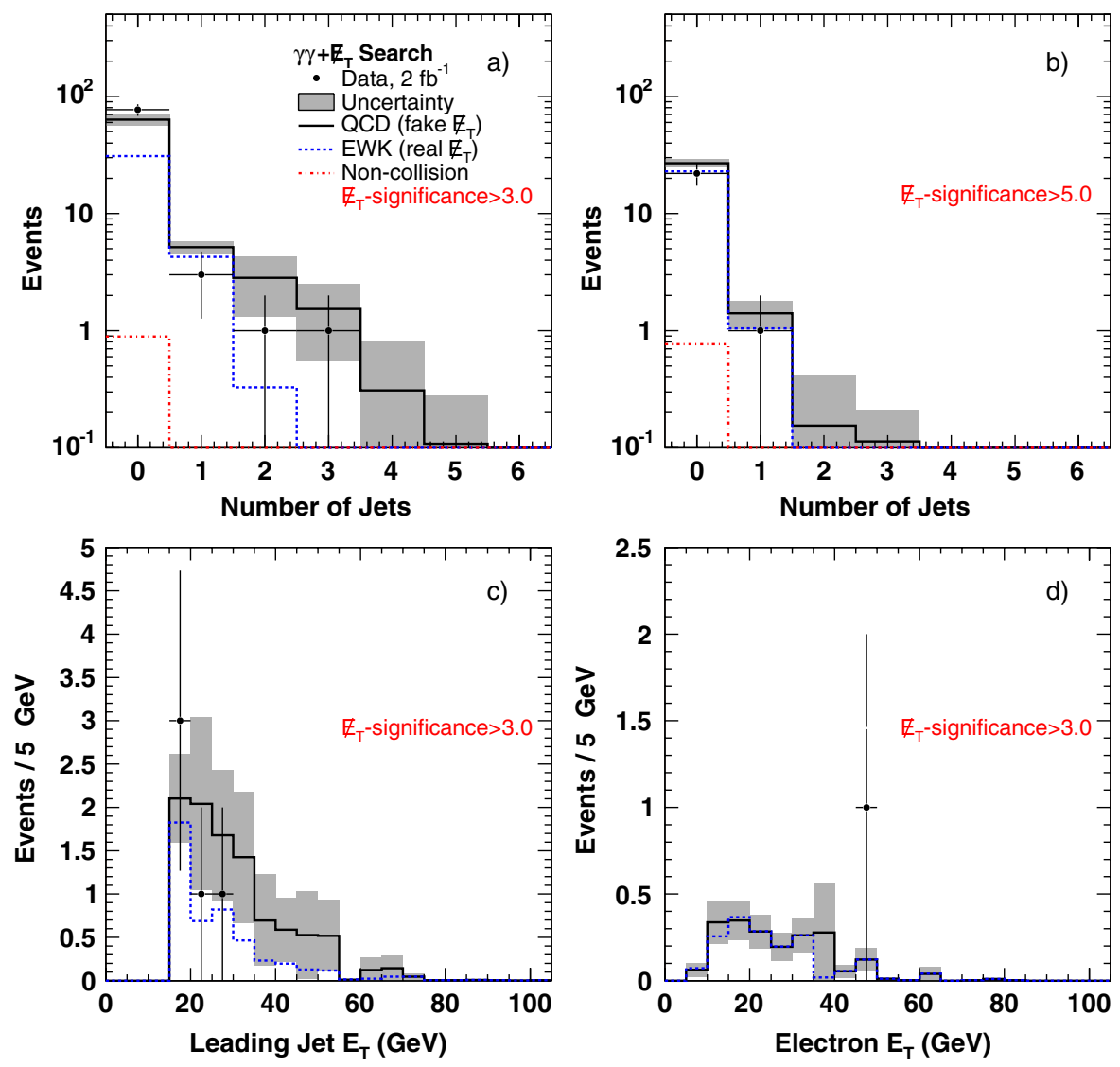

FIG. 10 (color online). Properties of additional objects in $\gamma \gamma+E_{T}$ candidate events: (a) the multiplicity of jets with $E_{T}>15$ GeV for events with $E_{T}$-significance $>3$; (b) the multiplicity of jets with $E_{T}>15 \mathrm{GeV}$ for events with $E_{T}$-significance $>5$; (c) the leading jet $E_{T}$ for events with $E_{T}$-significance $>3$; and (d) the electron $E_{T}$ for events with $E_{T}$-significance $>3$. In all figures, the data (marker) is compared with the total background prediction (solid line with the gray band representing the total uncertainty). The total background prediction is a sum (shown by the stacked histograms) of the QCD, electroweak (dashed line), and noncollision [dasheddotted line on (a) and (b) only] backgrounds.

predicted background shapes for all studied kinematic distributions that are expected to be sensitive to production of new particles.

In summary, we have searched for anomalous production of $\gamma \gamma+E_{T}$ events in data corresponding to $2.0 \mathrm{fb}^{-1}$ of integrated luminosity. No significant deviations from the SM background predictions are observed.

\section{CONCLUSIONS}

We performed a model-independent search for anomalous production of two photons with an electron, muon, $\tau$ lepton, or large missing transverse energy. The analysis of a $\gamma \gamma+e / \mu$ signature was performed using data corresponding to $1.1 \mathrm{fb}^{-1}$ of integrated luminosity. After final selection, we observed one $\gamma \gamma+e$ candidate event and zero $\gamma \gamma+\mu$ events, in agreement with the expected background of $3.79 \pm 0.54$ and $0.71 \pm 0.10$ events, respectively. The kinematic properties of the $\gamma \gamma+e$ event were consistent with the SM predictions. The silicon-track rejection technique applied in this search allows for more than $60 \%$ reduction in the bremsstrahlung background (the dominant background in the electron channel) and has a promising potential for future searches with the $\gamma+e+X$ signature.

The search for new physics in $\gamma \gamma+\tau$ was based on data corresponding to $2.0 \mathrm{fb}^{-1}$ of integrated luminosity. We observed 34 data events, in good agreement with the expected background of $46 \pm 10$ events. The kinematic distributions of the selected events did not reveal any deviations from the SM predictions.

The study of the $\gamma \gamma+E_{T}$ signature was performed using data from $2.0 \mathrm{fb}^{-1}$ of integrated luminosity. The events of interest were selected based on the $E_{T}$-significance, rather than a fixed $E_{T}$-cut. This method proved to be very effective in rejecting events with fake $E_{T}$, while remaining sensitive to new physics processes even with moderate values of $E_{T}\left(E_{T} \sim 20-40 \mathrm{GeV}\right)$. We selected 82, 31, and 23 data events with the $E_{T}$-significance greater than 3, 4, and 5, respectively. These results are consistent with the expected SM background of $71.7 \pm$ $7.5,39.0 \pm 3.1$, and $30.4 \pm 2.4$ events, respectively. 
The examined kinematic distributions for the observed events with $E_{T}$-significance greater than three and five are in a good agreement with the predicted background shapes. The METMODEL developed as part of the $\gamma \gamma+E_{T}$ search was also successfully applied to suppress multijet background with fake $E_{T}$ in the first observation of vector boson pairs in a final state with two jets and $E_{T}$ at the Tevatron [41]. Finally, the reported in this paper modelindependent analysis was later used as a basis for a search for supersymmetry with gauge-mediated breaking in $\gamma \gamma+$ $E_{T}$ events [42]. The data samples used in these two analyses have a $60 \%$ overlap.

In summary, no significant deviations from the standard model were observed in the numbers of recorded events and their kinematic properties in signatures with two photons and an additional electron, muon, $\tau$ lepton, or large $E_{T}$. We also did not observe any new ee $\gamma \gamma+E_{T}$ candidate events, similar to the one reported in Ref. [9]. With improved analysis techniques and up to 20 times more data compared to the previous searches [9-11], this modelindependent search is substantially more sensitive to new physics.

\section{ACKNOWLEDGMENTS}

We thank the Fermilab staff and the technical staffs of the participating institutions for their vital contributions. This work was supported by the U.S. Department of Energy and National Science Foundation; the Italian Istituto Nazionale di Fisica Nucleare; the Ministry of Education, Culture, Sports, Science and Technology of Japan ; the Natural Sciences and Engineering Research Council of Canada; the National Science Council of the Republic of China; the Swiss National Science Foundation; the A.P. Sloan Foundation; the Bundesministerium für Bildung und Forschung, Germany; the World Class University Program, the National Research Foundation of Korea; the Science and Technology Facilities Council and the Royal
Society, UK; the Institut National de Physique Nucleaire et Physique des Particules/CNRS; the Russian Foundation for Basic Research; the Ministerio de Ciencia e Innovación, and Programa Consolider-Ingenio 2010, Spain; the Slovak R\&D Agency; and the Academy of Finland.

\section{APPENDIX A: DEFINITIONS}

\section{Diphoton triggers}

There are two diphoton paths in the CDF three-level trigger: the first path requires two isolated electromagnetic clusters with $E_{T}>12 \mathrm{GeV}$ (diphoton-12), and the second path requires two electromagnetic clusters with $E_{T}>$ $18 \mathrm{GeV}$ and has no isolation requirement (diphoton-18). The transverse energy of clusters is calculated with respect to the nominal center of the detector at $z=0 \mathrm{~cm}$. The trigger requirements at each level are briefly described below.

At Level-1, events with two towers with EM $E_{T}>$ $8 \mathrm{GeV}$ each are required. For each trigger tower, the amount of energy in the hadronic compartment of the calorimeter $\left(E^{\mathrm{HAD}}\right)$ has to be consistent with that of an electromagnetic object. A trigger tower consists of two adjacent towers in the same calorimeter wedge, so that the granularity is approximately $\Delta \eta \times \Delta \phi \simeq 0.2 \times 15^{\circ}$.

The Level-2 requirements are different for the two triggers. The diphoton-12 trigger selects events if there are two isolated clusters (seeds) with EM $E_{T}>10 \mathrm{GeV}$ each. The isolation (ISO) energy is calculated as a sum of the transverse energy in nine towers surrounding the seed tower according to five preset patterns. The ISO energy in each of the patterns has to be less than $3 \mathrm{GeV}$ or $15 \%$ of the seed energy, whichever is larger. The diphoton-18 trigger requires two towers with $\mathrm{EM} E_{T}>16 \mathrm{GeV}$ each at Level-2.

The events are fully reconstructed at Level-3. At this level, for both triggers, the energy profile at the shower maximum $\left(\chi_{\text {CES }}^{2}\right)$ of each photon candidate has to be consistent with that of a single photon. The diphoton-12

TABLE IV. Summary of the diphoton trigger requirements.

\begin{tabular}{lcc}
\hline \hline Trigger level & Diphoton-12 & Diphoton-18 \\
\hline Level-1 & $\mathrm{EM} E_{T}>8 \mathrm{GeV}$ & same \\
& $E^{\mathrm{HAD}} / E^{\mathrm{EM}}<0.125$ & same \\
& $N_{\text {cluster }}=2$ & same \\
EM $E_{T}>10 \mathrm{GeV}$ & $\mathrm{EM} E_{T}>16 \mathrm{GeV}$ \\
& $E^{\mathrm{HAD}} / E^{\mathrm{EM}}<0.125$ & same \\
& $E_{T}^{\mathrm{ISO}}<3 \mathrm{GeV}$ or $E_{T}^{\mathrm{ISO}} / E_{T}<0.15$ & N/A \\
& $N_{\text {cluster }}=2$ & same \\
Level-3 & $\mathrm{EM} E_{T}>12 \mathrm{GeV}$ & $\mathrm{EM} E_{T}>18 \mathrm{GeV}$ \\
& $E^{\mathrm{HAD}} / E^{\mathrm{EM}}<0.055+0.00045 \times E / \mathrm{GeV}$ if $E<200 \mathrm{GeV}$ & same \\
& $E_{T}^{\mathrm{ISO}}<2 \mathrm{GeV}$ or $E_{T}^{\mathrm{ISO}} / E_{T}<0.1$ & N/A \\
& shower profile: $\chi_{\mathrm{CES}}^{2}<20$ & same \\
& $N_{\text {cluster }}=2$ & same \\
\hline \hline
\end{tabular}


trigger selects events with two isolated photon candidates with $E_{T}>12 \mathrm{GeV}$. The isolation energy at the level-3 is calculated as the sum of $E_{T}$ in all towers (except for photon towers) within the cone of $\Delta R=\sqrt{\Delta \eta^{2}+\Delta \phi^{2}}<0.4$ centered around the photon candidate. This ISO energy has to be less than $2 \mathrm{GeV}$ or $10 \%$ of the photon energy, whichever is larger. The diphoton-18 trigger has no isolation requirement and accepts events with two photon candidates with $E_{T}>18 \mathrm{GeV}$. Table IV gives a summary of all trigger requirements for events with EM objects in the central calorimeter.

\section{Photon identification}

Photon candidates have to satisfy strict (also referred to as "tight") photon identification requirements. The EM cluster has to be located inside the well-instrumented region of the CES chamber, away from the $\phi$-boundary of a calorimeter tower [43]. The energy deposition pattern in both transverse profiles at CES has to be consistent with that of an electromagnetic object. The ratio of the energy measured in the HAD calorimeter to the EM energy, $E^{\mathrm{HAD}} / E^{\mathrm{EM}}$, has to satisfy the $E^{\mathrm{HAD}} / E^{\mathrm{EM}}<0.055+$ $0.00045 \times E^{\gamma}$ requirement. To distinguish photons from electrons, no high- $p_{T}$ charged track should point into the cluster $\left(N_{\text {track }} \leq 1\right.$ with track $\left.p_{T}<1.0+0.005 \times E_{T}\right)$. The main sources of fake photons are $\pi^{0}$ and $\eta^{0}$ produced in jets. These mesons are usually produced in association with other particles. To reduce this contamination from jets, the photon candidate must be isolated in the calorimeter and tracking chamber. To calculate the calorimeter isolation (cal-ISO), the $E_{T}$ deposited in the calorimeter towers within the cone of $\Delta R<0.4$ around the EM cluster is summed, and the $E_{T}$ due to the EM cluster is subtracted. The cal-ISO is then corrected for the photon's energy leakage into towers in the neighboring wedge and for the contribution from multiple interactions in the same bunch crossing. The track isolation (track-ISO) is calculated as $\sum p_{T}$ of tracks inside a cone $\Delta R<0.4$ and satisfying $\left|z_{\text {vertex }}-z_{\text {track }}\right|<5 \mathrm{~cm}$. Both cal-ISO and track-ISO must be consistent with the amount of energy expected from the underlying event. In addition to calorimeter and tracking isolation, there should be no other significant energy $\left(E_{T}\right.$ of 2nd CES cluster) deposited in the CES chamber containing the photon candidate. Table $\mathrm{V}$ provides a summary of the photon identification requirements described above.

We obtain the $\gamma \gamma$ control sample by selecting events where two photon candidates pass relaxed (loose) photon identification requirements, but at least one of them fails the tight cuts. The main difference between loose and tight photon requirements is in the amount of allowed isolation energy (see Table V). The resulting $\gamma \gamma$ control sample is dominated by jet $-\gamma$ and jet-jet events where one or both photon candidates are faked by jets. The fraction of real $\gamma \gamma$ events in the control sample is only $5 \%$.

In addition to the photon identification requirements described above, we also apply cuts to remove contamination from noncollision sources. Muons produced in the beam halo are known to fake a photon signature [40]. These energetic muons travel parallel to the beam pipe and deposit energy in many towers of one wedge, consistent with a minimum ionizing particle. When the muon undergoes energetic bremsstrahlung, it may also create one or two fake photon candidates. Probability for a single photon $\mathrm{BH}$ event to overlap with a collision event with a photon candidate is very low. Therefore, events with both fake photons from one muon are a dominant source of the $\mathrm{BH}$ background. We use this fact to suppress such $\mathrm{BH}$ events. We reject events if $\Delta \phi_{\gamma \gamma}<0.524 \mathrm{rad}$ and if there are more than two hadronic and four central electromagnetic towers above $0.1 \mathrm{GeV}$ threshold [44]. The efficiency of these requirements for collision events is estimated with data $Z \rightarrow e^{+} e^{-}$events and found to be $\sim 100 \%$. The rejection power of the cuts for beam halo events is found to be $90.4 \% \pm 0.2 \%$, as estimated using a very pure sample of beam halo events with two photon candidates located in the same calorimeter wedge. The criteria used to select this sample are discussed in detail in Ref. [40].

Muons from cosmic rays may also bremsstrahlung in the calorimeter and create fake photon candidates. To suppress

TABLE V. Summary of the standard (tight) and relaxed (loose) photon identification requirements for the signal and control $\gamma \gamma$ samples, respectively.

\begin{tabular}{lcc}
\hline \hline Cuts & Tight photon ID & Loose photon ID \\
\hline$E_{T}^{\gamma}$ & $\geq 13 \mathrm{GeV}$ & same \\
Shower profile in CES: $\chi^{2}$ & $\leq 20$ & same \\
$E^{\mathrm{HAD}} / E^{\mathrm{EM}}$ & $\leq 0.055+0.00045 \times E / \mathrm{GeV}$ & $\leq 0.125$ \\
Cal-ISO & $\leq 0.1 \times E_{T}$ if $E_{T}<20 \mathrm{GeV}$ or & $\leq 0.15 \times E_{T}$ if $E_{T}<20 \mathrm{GeV}$ or \\
& $\leq 2.0 \mathrm{GeV}+0.02 \times\left(E_{T}-20 \mathrm{GeV}\right)$ & $\leq 3.0 \mathrm{GeV}+0.02 \times\left(E_{T}-20 \mathrm{GeV}\right)$ \\
Track-ISO & $\leq 2.0 \mathrm{GeV}+0.005 \times E_{T}$ & $\leq 5 \mathrm{GeV}$ \\
$N_{\text {tracks }}$ in cluster & $\leq 1.0 \mathrm{GeV}+0.005 \times E_{T}$ & $\mathrm{same}$ \\
Track $p_{T}$ if $N_{\text {tracks }}=1$ & $\leq 0.14 \times E_{T}$ if $E_{T}<18 \mathrm{GeV}$ & $\leq 0.25 \times E_{T}$ \\
$E_{T}$ of 2 nd CES cluster & $\leq 2.4 \mathrm{GeV}+0.01 \times E_{T}$ if $E_{T} \geq 18 \mathrm{GeV}$ & no cut \\
&
\end{tabular}


these events, we use different approaches for data collected before and after the timing system in the EM calorimeter [24] was installed. In the first $0.44 \mathrm{fb}^{-1}$ of integrated luminosity for which the EM timing is not available, we reject events if there is a segment of hits ("stub") in the muon drift chambers within a cone of $30^{\circ}$ around the direction of any of the photon candidates that is not linked to a track in the COT (trackless muon stub). This requirement rejects approximately $85 \%$ of cosmic rays and is approximately $98 \%$ efficient for $\gamma \gamma$ events. In data from the later $1.6 \mathrm{fb}^{-1}$ of integrated luminosity for which the EM timing is available, we reject events if one of the photon candidates has arrival time $\left|T_{1,2}\right|>4 \sigma_{T}$ or two photons have $|\Delta T|=\left|T_{1}-T_{2}\right|>4 \sigma_{\Delta T}$, where $\sigma_{T}=$ $1.67 \mathrm{~ns}$ and $\sigma_{\Delta T}=1.02 \mathrm{~ns}$ are the timing resolutions obtained by studying the EM timing of electrons from $Z \rightarrow$ $e^{+} e^{-}$events [45]. These EM timing requirements reject 99.4\% of cosmic rays, while they are $99.9 \%$ efficient for prompt $\gamma \gamma$ events.

Another source of fake photons is electrons from electroweak processes which are misreconstructed as prompt photons. This occurs when either an electron undergoes a catastrophic bremsstrahlung in the detector material in front of the COT or when its track does not get reconstructed. In both cases, electrons usually leave a few hits in the silicon detectors and their tracks can be partially recovered by a special tracking algorithm [34]. This algorithm looks for silicon hits along two helix curves connecting vertex and EM cluster positions. The helix curvature is uniquely defined by the EM cluster $E_{T}$, and two curves correspond to a positive and negative charge hypotheses. If any of the photon candidates is matched to such a track, we reject the event. This technique is used in the $\gamma \gamma+e / \mu$ and $\gamma \gamma+E_{T}$ searches and it is referred to as the "silicon-track rejection" in the main text.

\section{Electron identification}

We select electrons using the CDF standard criteria. An electron is characterized by a narrow shower in the electromagnetic calorimeter and a matching track (either in the COT or silicon detector) originating from the primary vertex. The transverse EM energy, $E_{T}^{\mathrm{EM}}$, must be greater than $20 \mathrm{GeV}$. The $E^{\mathrm{HAD}} / E^{\mathrm{EM}}$ ratio has to be less than 0.055 . The lateral energy distribution of the shower must be consistent with that for an electron. Candidates are required to be isolated in the calorimeter and to contain at least $90 \%$ of the total transverse energy within a cone of $\Delta R=0.4$. For an electron detected in the central region $(|\eta|<1.05)$, the matching track is required to be well reconstructed by the COT and have $p_{T}>10 \mathrm{GeV} / c$. The ratio of the electron energy to track momentum, $E / p$, must be less than 2.0. Electrons from photon conversions are suppressed by rejecting the candidates which have an oppositely charged track with a small separation in the $x y$ plane and a minimal difference in the polar angle. For an electron detected in the forward region $(1.2<|\eta|<2.0)$, the matching track is required to have a minimum of three hits measured in the silicon detector. We do not apply further requirements on the forward matching tracks because fewer measurements per track are available and the momentum measured in the forward region is not as reliable as that measured in the central region. More details of electron identification can be found in Ref. [46].

\section{Muon identification}

We select muons using the CDF standard criteria. A muon is characterized by a well-reconstructed COT track which is matched to track segments (stubs) in the central muon detectors, and an energy deposition in the EM and HAD calorimeters consistent with a minimum ionizing particle. The $p_{T}$, measured either with the COT only, or with the COT and the silicon detector if the silicon hits are available, must be greater than $20 \mathrm{GeV} / c$. Two types of muons are selected: CMUP $(|\eta|<0.6)$ and CMX $(0.6<$ $|\eta|<1.0)$. The CMUP muon candidate requires a match between the track and the stubs in the CMU and CMP detectors. The CMX muon candidate requires a match to a muon stub in the CMX detector. In order to reduce the background from cosmic rays or hadrons which decay in flight, we require the track to be consistent with originating along the beam line. Cosmic muons are further suppressed via their back-to-back track topology and asynchronous timing measured in the COT. More details on the muon identification can be found in Ref. [46].

\section{Tau-lepton identification}

The $\tau$ lepton has a $\sim 18 \%$ branching fraction for decays into an electron or muon, with neutrinos. When this occurs, the event would be categorized in the $e / \mu$ final state and addressed in that study. In the $\tau$ lepton search we address only the hadronic decay modes.

To identify the hadronic decays of $\tau$ leptons [47], we require a narrow cluster of one or three tracks and calorimeter energy. This cluster must be consistent with a $\tau$ lepton in several ways, inconsistent with an electron, and isolated from other nearby calorimeter energy.

The clustering begins with a single tower with $E_{T}>$ $6 \mathrm{GeV}$. Up to five more towers may be added to the cluster if they are adjacent and have $E_{T}>1 \mathrm{GeV}$. At least one high-quality track with $p_{T}>6 \mathrm{GeV} / c$ must be associated with the cluster. This track defines the origin point of the $\tau$ lepton. The cone subtending an angle of $\theta_{\text {sig }}$ from the track direction defines the signal region where the $\tau$ lepton decay products are expected. This angle is fixed to be 0.17 at low $\tau$ lepton $E_{T}$ and is smaller for $E_{T}>30 \mathrm{GeV}$, shrinking to 0.05 at $E_{T}=100 \mathrm{GeV}$, allowing for greater rejection as the $\tau$ lepton decay products become highly collimated. A second cone given by $\theta<0.52$ defines an isolation annulus. The calorimeter and the shower maximum detector are used to define $\pi^{0}$ candidates in the $\tau$ lepton signal cone and 
the isolation annulus. To reject electrons some hadronic energy, consistent with the observed signal-cone tracks, is required.

The $\tau$ lepton four-vector is defined by the total fourvector of the tracks and $\pi^{0}$ candidates in the signal cone. If the calorimeter cluster energy is significantly greater than this sum, the calorimeter cluster energy is used instead. The "visible" mass of the $\tau$ lepton is found as the magnitude of this total four-vector.

We define two levels of $\tau$ lepton identification: a loose identification (used in studies and background techniques) and the standard or tight identification, used for the signal region search.

Apart from the selection included in the reconstruction as described above, the loose identification requires only $E_{T}>15 \mathrm{GeV}$. The tight selection also requires visible $\tau$ lepton mass less than $1.8 \mathrm{GeV}$, total track $p_{T}$ in the isolation cone less than $1.0 \mathrm{GeV}, \pi^{0} E_{T}$ in the isolation cone less than $0.6 \mathrm{GeV}$, and one or three tracks in the signal cone, with total charge of \pm 1 .

\section{Jets}

We reconstruct jets by using the cone clustering algorithm [48] with a cone radius $R=\sqrt{\Delta \phi^{2}+\Delta \eta^{2}}=0.4$. Identified photons and electrons are removed from a list of jets. The jet energy is corrected for a nonlinearity of the detector response and for contributions due to underlying event and multiple interactions in the same bunch crossing [49]. Unless otherwise stated, we only consider jets with $E_{T}>15 \mathrm{GeV}$ and $|\eta|<3.0$.

\section{Missing transverse energy and $H_{T}$}

The missing transverse energy, $E_{T}$, is defined as an energy imbalance in the calorimeter and it is a signature of neutrinos or new particles that do not interact with the detector material. The $E_{T}$ is calculated from all calorimeter towers with $E_{T}>0.1 \mathrm{GeV}$ in the region $|\eta|<3.6$ according to $\vec{E}_{T}=-\sum_{i} E_{T}^{i} \vec{n}_{i}$ where $\vec{n}_{i}$ is a unit vector that points from the interaction vertex to the $i$ th calorimeter tower in the transverse plane. To improve resolution and reduce the number of events with large fake $E_{T}$, we apply corrections to the $E_{T}$ to account for a nonlinearity of the detector response for jets with $E_{T}>15 \mathrm{GeV}$ and for presence of reconstructed muons, which do not deposit their total energy in the calorimeter.

One of the global kinematic characteristics of any hard scattering process is the total transverse energy of final products, $H_{T}$. We define $H_{T}$ for each event as a sum of the transverse energies of all identified objects: photons, electrons, muons, visible energy of $\tau$ leptons, jets, and $E_{T}$. According to many theoretical models, new physics is expected to appear at large energy scales and may reveal itself in an anomalous rate of events with large values of $H_{T}$.

\section{Unclustered energy}

The activity due to the underlying event and additional interactions in the same bunch crossing is characterized by the soft unclustered energy, $\sum^{\text {soft }} E_{T}$. We calculate $\sum^{\text {soft }} E_{T}$ for each event by taking the difference between the total transverse energy in the event and transverse energies of all reconstructed photons, electrons, and jets: $\sum^{\text {soft }} E_{T}=$ $\sum^{\mathrm{all}} E_{T}-\sum E_{T}^{\mathrm{jet}}-\sum E_{T}^{\gamma}-\sum E_{T}^{\mathrm{ele}}$.

\section{The e $\gamma$ events in $\gamma \gamma+E_{T}$ analysis}

We use inclusive $e \gamma$ events to obtain a data/MC normalization factor for the MC-based estimate of the EWK backgrounds in the search for anomalous production of $\gamma \gamma+E_{T}$ events. To minimize differences between $e \gamma$ and $\gamma \gamma$ samples, we obtain the $e \gamma$ events in data and MC by using the same diphoton triggers (for data) and analysis selection procedures as used to derive our $\gamma \gamma$ baseline sample. In this selection, we treat an electron as a photon (i.e., we apply the same cuts as in Table V) with only one exception: we also require the presence of a track pointing to an EM cluster. This track must satisfy the $0.8<$ $E / p<1.2$ requirement where $E$ is the energy of the EM cluster and $p$ is the track momentum. All additional tracks must pass the cuts listed in Table V.

\section{APPENDIX B: FAKE RATES}

\section{The $e \rightarrow \gamma$ fake rate}

Electrons may be misidentified as signal photons due to hard bremsstrahlung in the detector material, inefficiency of track reconstruction, or collinear final-state radiation. We measure the misidentification probability, $\mathcal{P}(e \rightarrow \gamma)$, using Drell-Yan $Z / \gamma^{*}$ events. The $\mathcal{P}(e \rightarrow \gamma)$ is defined as the ratio of the number of reconstructed $Z / \gamma^{*} \rightarrow e \gamma$ events to the number of reconstructed $Z / \gamma^{*} \rightarrow e e$ events. The $E_{T}$ dependence of $\mathcal{P}(e \rightarrow \gamma)$ is obtained from the simulation. The overall normalization is scaled by the ratio of data-toMC probabilities measured at $E_{T}=40-50 \mathrm{GeV}$, around the $Z$ peak. The electrons have been selected using two types of identification: (1) standard central-electron criteria in Sec. A 3, (2) photonlike electron criteria in Sec. A 9. The result from identification- 1 has been used in the $\gamma \gamma e$ and $\gamma \gamma \mu$ searches, while the result from identification- 2 has been used in the cross-checks of $\gamma \gamma e, \gamma \gamma \mu$, and $\gamma \gamma E_{T}$ searches. The $\mathcal{P}(e \rightarrow \gamma)$ for identification-1, before $\left[\mathcal{P}(e \rightarrow \gamma)^{B}\right]$ and after $\left[\mathcal{P}(e \rightarrow \gamma)^{A}\right]$ applying silicon-track rejection, is measured with the data and MC Drell-Yan samples and parameterized as a function of the electron $E_{T}$ (in $\mathrm{GeV}$ ):

$$
\begin{aligned}
& \mathcal{P}(e \rightarrow \gamma)^{\mathrm{B}}=S^{\mathrm{B}} \cdot\left(e^{-2.991-0.045 \cdot E_{T}}+0.007\right), \\
& \mathcal{P}(e \rightarrow \gamma)^{\mathrm{A}}=S^{\mathrm{A}} \cdot \mathcal{P}(e \rightarrow \gamma)^{\mathrm{B}} \cdot\left(1-\epsilon\left(E_{T}\right)\right),
\end{aligned}
$$



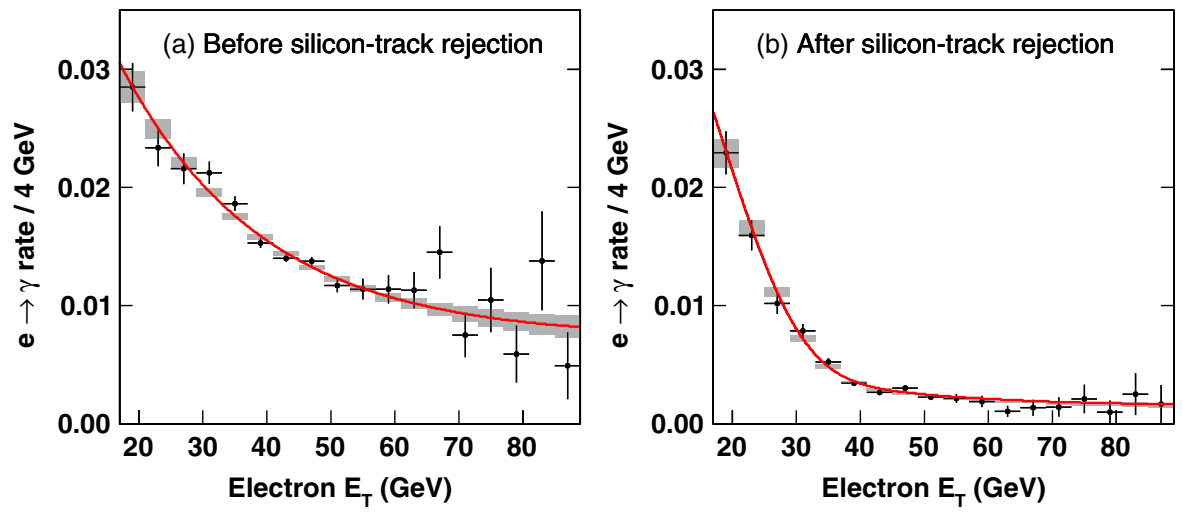

FIG. 11 (color online). Probability for a CDF standard central electron to be misidentified as a standard central photon as measured in the Drell-Yan MC, before (a) and after (b) applying the silicon-track rejection. The misidentification probabilities (points) are parameterized as a function of electron $E_{T}$ at the parton level. The gray boxes indicate the systematic uncertainties in each $E_{T}$ bin due to the uncertainties on fit parameters.

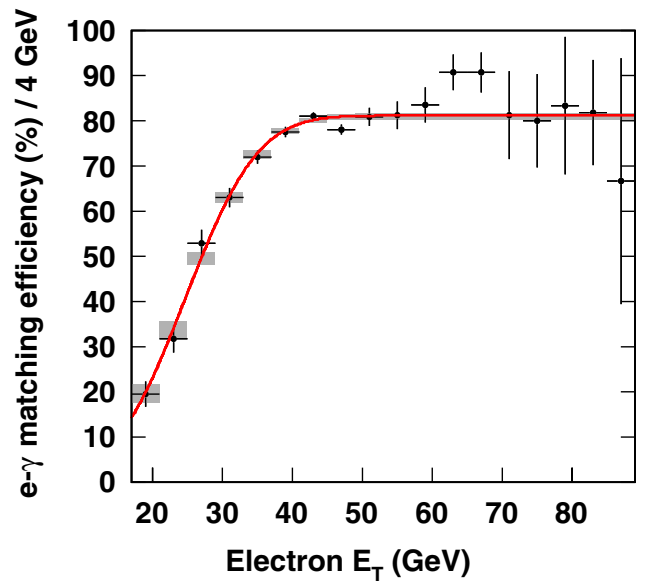

FIG. 12 (color online). Efficiency for an electron reconstructed as a CDF standard central photon to be matched to the siliconelectron track, as a function of electron $E_{T}$ at the parton level, measured in the Drell-Yan MC. The gray boxes indicate the systematic uncertainties in each $E_{T}$ bin due to the uncertainties on fit parameters.

where $S^{\mathrm{B}}$ and $S^{\mathrm{A}}$ are the data-to-MC scaling factors:

$$
S^{\mathrm{B}}=1.08 \pm 0.09, \quad S^{\mathrm{A}}=1.32 \pm 0.20,
$$

and the $\epsilon\left(E_{T}\right)$ is the efficiency of silicon-track rejection measured with the Drell-Yan MC:

$$
\begin{aligned}
& \epsilon\left(E_{T}\right)=0.405 \cdot \frac{2}{\sqrt{\pi}} \int_{z}^{\infty} e^{-t^{2}} d t, \\
& z=0.086 \cdot\left(24.711-E_{T}\right) .
\end{aligned}
$$

The electron $E_{T}$ at the parton level is further translated to the photon $E_{T}$ at the reconstruction level using a simulation study. Figure 11 shows the $\mathcal{P}(e \rightarrow \gamma)^{\mathrm{B}}$ and $\mathcal{P}(e \rightarrow \gamma)^{\mathrm{A}}$ measured in the Drell-Yan MC, without data-to-MC scaling factors applied. Figure 12 shows the $\epsilon\left(E_{T}\right)$. The average $\mathcal{P}(e \rightarrow \gamma)^{\mathrm{B}}$ is about $1.5 \%$ with an $11 \%$ fractional uncertainty, and the average $\mathcal{P}(e \rightarrow \gamma)^{\mathrm{A}}$ is about $0.4 \%$ with a $17 \%$ fractional uncertainty. The uncertainties come from the limited size of $Z$ data sample which determines the data-to-MC scaling factor, the variation of fitting methods which determines the number of $Z$ candidates, and the difference between results measured in the diphoton and inclusive electron triggers.

\section{The jet $\rightarrow \gamma$ fake rate}

Hadrons in jets, such as $\pi^{0}, \eta^{0}$, and $K_{s}^{0}$, may decay into multiple photons. The segmentation of the CDF EM calorimeter is not sufficiently small to separate these photons and the standard reconstruction algorithm will reconstruct these hadron daughters as a single photon candidate. The probability to misidentify a jet as a signal photon, $\mathcal{P}($ jet $\rightarrow$ $\gamma$ ), has been measured in Ref. [31], using data collected with inclusive jet triggers. The $\mathcal{P}($ jet $\rightarrow \gamma)$ is defined as the number of identified photon candidates times the fake photon fraction $\left(F_{\mathrm{QCD}}\right)$ and divided by the number of jets. The fraction $F_{\mathrm{QCD}}$ is required because the identified photon candidates in the jet data will contain real photons not relevant to the fake rate. Reference [31] has determined $F_{\mathrm{QCD}}$ statistically by combining the following information: (a) the lateral shower shape measured in the wire and strip chamber (CES), (b) the extra energy in a cone of $\Delta R=0.4$ around the photon candidate (cal-ISO) measured in the calorimeters, and (c) the conversion rate measured in the central preshower detector. The $\mathcal{P}($ jet $\rightarrow \gamma)$ is parameterized as a function of the jet $E_{T}$ (in $\mathrm{GeV}$ ) and found to be

$$
\mathcal{P}(\text { jet } \rightarrow \gamma)=10^{-3} \cdot\left(e^{2.397-0.153 \cdot E_{T}(\mathrm{jet})}+0.404\right) .
$$

The fake photon $E_{T}$ is smaller than the original jet $E_{T}$ because the fake photon is often accompanied by other particles from that jet. The translation of the jet $E_{T}$ to the photon $E_{T}$ has been studied using simulations and is 
represented by a Gaussian distribution with a mean of 0.937 and a width of 0.048 . The $\mathcal{P}($ jet $\rightarrow \gamma)$ is about $0.2 \%$ at $E_{T}^{\gamma}=13 \mathrm{GeV}$ and $0.04 \%$ for $E_{T}^{\gamma}>50 \mathrm{GeV}$, with a systematic uncertainty ranging from $50 \%$ to $200 \%$. The sources of systematic uncertainties include the differences between the methods for determining $F_{\mathrm{QCD}}$, the differences of results when using a loose photon candidate as the fake denominator, variation of the mixture of quark jets and gluon jets, and variation of fragmentation model in the simulation which changes the Gaussian function of $E_{T}$ translation.

\section{The jet $\rightarrow e / \mu$ fake rate}

Hadrons in jets may be misidentified as electrons due to inelastic charge exchange or the production of an energetic conversion electron. The inelastic charge exchange in the EM calorimeter, $\pi^{-} p \rightarrow \pi^{0} n$ or $\pi^{+} n \rightarrow \pi^{0} p$, results in a track in the COT due to the $\pi^{ \pm}$and an EM shower in the calorimeter due to the photons from $\pi^{0}$ decay. The combination of a charged track and an EM shower gives a fake electron candidate. Hadrons can also decay into muons before interacting with the calorimeter (e.g., $K^{+} \rightarrow$ $\mu^{+} \nu_{\mu}$ ) or pass through the calorimeter into the muon chamber (punch through) with minimal interaction and give fake muon candidates. The probability to misidentify a jet as an electron or a muon, $\mathcal{P}($ jet $\rightarrow e, \mu)$, has been measured in Ref. [33], using data collected with inclusive jet triggers. $\mathcal{P}($ jet $\rightarrow e, \mu)$ is defined as the ratio of the number of identified electron/muon candidates to the number of "fakeable" objects (denominator). The fakeable object is a jet with uncorrected $E_{T}>4 \mathrm{GeV}$ for central electrons, a jet with uncorrected $E_{T}>15 \mathrm{GeV}$ for forward electrons, and an isolated track with $p_{T}>4 \mathrm{GeV} / c$ and minimal extra energy in the cone of $\Delta R=0.4$ for muons. A track is considered to be isolated if the total $E_{T}$ of calorimeter towers within the cone of $\Delta R<0.4$ around the track is less than $4 \mathrm{GeV}$ or less than $10 \%$ of the track's momentum. The misidentification probabilities, parameterized as a function of the jet $E_{T}$ (in $\mathrm{GeV}$ ), for the electrons are

$$
\begin{aligned}
& \mathcal{P}\left(\text { jet } \rightarrow e^{\text {central }}\right)=0.00013+e^{-7.940-0.194 \cdot E_{T}(\text { jet }),} \\
& \mathcal{P}\left(\text { jet } \rightarrow e^{\text {forward }}\right)=0.00032+0.000012 \cdot E_{T}(\text { jet }) .
\end{aligned}
$$

The translation of the jet $E_{T}$ to the electron $E_{T}$ has been studied using simulations. The ratio of electron $E_{T}$ to jet $E_{T}$ is represented by a Gaussian distribution with a mean of 0.89 and a width of 0.06 . The misidentification probabilities, parameterized as a function of the track $p_{T}$ (in $\mathrm{GeV} / c$ ), for the muons are

$$
\begin{aligned}
& \mathcal{P}\left(\text { track } \rightarrow \mu^{\mathrm{CMUP}}\right)=0.00086+0.00017 \cdot p_{T}(\mu), \\
& \mathcal{P}\left(\operatorname{track} \rightarrow \mu^{\mathrm{CMX}}\right)=0.00082+0.00020 \cdot p_{T}(\mu) .
\end{aligned}
$$

Since the misidentification probabilities were measured up to $E_{T}=50 \mathrm{GeV}$ and $p_{T}=50 \mathrm{GeV} / c$ and the misidentification probabilities are expected to reach a plateau, we assign a constant value to all misidentification probabilities for $E_{T} \geq 50 \mathrm{GeV}$ and $p_{T} \geq 50 \mathrm{GeV} / c$. The $\mathcal{P}($ jet $\rightarrow$ $e, \mu)$ averages $\approx 0.01 \%$ for central electrons, $\approx 0.04 \%$ for forward electrons, and $\approx 1.0 \%$ for central muons, with a $50 \%$ systematic uncertainty estimate provided by the variation of results measured in different jet triggers.

\section{The jet $\rightarrow \tau$ fake rate}

The probability of a quark or gluon jet to be misreconstructed as an hadronically decaying $\tau$ lepton is measured and then applied to a sample of jets to estimate the number of fake $\tau$ leptons we expect in that sample.

We measure this misidentification rate in a sample of inclusive jet triggers [50], using only the energy clusters for which the trigger is fully efficient. This jet sample has a negligible fraction of real $\tau$ leptons because the rates for $W / Z \rightarrow \tau+X$ and $c / b \rightarrow \tau+X$ processes are very small compared to the jet production rates. Therefore, the measurement of the misidentification rate is straightforward. We identify all loose and tight $\tau$ leptons (see Sec. A 5) and measure the rate by dividing the number of tight $\tau$ leptons by the number of loose $\tau$ leptons as a function of the $\tau$ candidate $E_{T}$.

We check the misidentification rate by using it to predict various distributions in the jet samples. We compare the number of $\tau$ leptons observed and predicted as a function of the first, second, and third jet $E_{T}$, the event total energy,

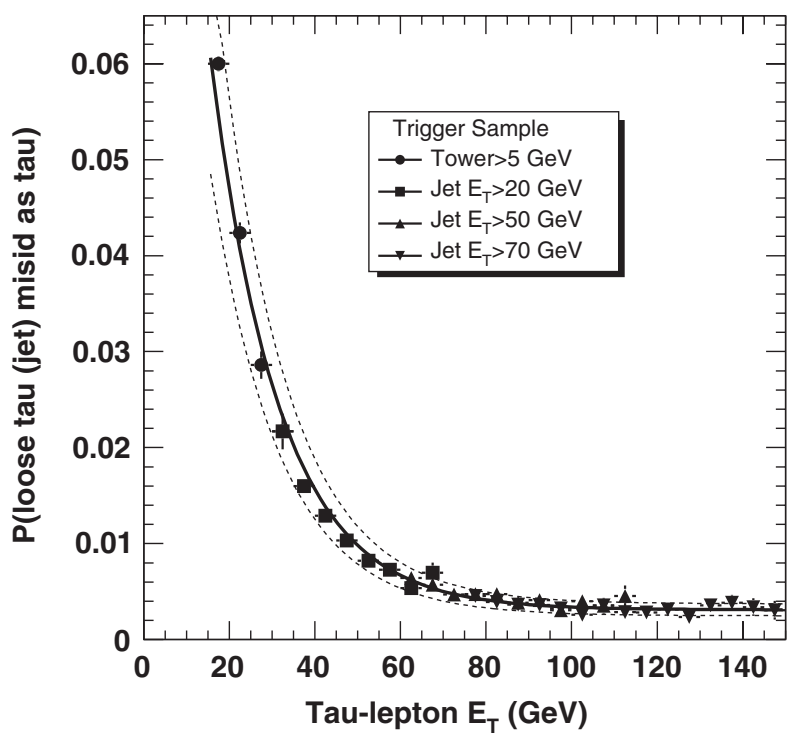

FIG. 13. The solid line represents the probability for objects passing loose $\tau$ lepton ID cuts to also pass tight $\tau$ lepton ID cuts (jet $\rightarrow \tau$ fake rate) as a function of $\tau$ lepton $E_{T}$, with the overlapping regions removed. The dashed lines are the systematic uncertainties on the jet $\rightarrow \tau$ fake rate $( \pm 1$ standard deviation). 
the underlying event energy, the number of jets, the number of interactions in the event, and the distance to the nearest jet. The only notable discrepancy is the case where the $\tau$ lepton is close to a second jet, where the jet's energy tends to spoil the $\tau$ lepton's isolation and reduce the fake rate. We include this effect in the application of the misidentification rate. The assigned systematic uncertainty of $20 \%$ accounts for any other discrepancies. The resulting function is shown in Fig. 13.

Finally, we identify the primary source of each jet in inclusive PYTHIA MC jet samples by searching for the highest- $E_{T}$ parton consistent with the jet direction. We then measure the misidentification rate in quark and gluon jets separately. We find the rate for gluon jets is approximately 3 times smaller than the rate for quark jets, which have a higher probability to fragment to a few energetic particles.

\section{APPENDIX C: THE $E_{T}$ RESOLUTION MODEL}

A major source of background in the $\gamma \gamma+E_{T}$ final state is diphoton candidate events with significant fake $E_{T}$ due to energy mismeasurement in the calorimeter. Given the large production rates for QCD processes $(\gamma \gamma, \gamma-$ jet, and jet-jet), fluctuations in energy measurements can result in a considerable fraction of such events. We predict the shape of this fake $E_{T}$ and calculate its significance on an event-by-event basis by means of the $E_{T}$ resolution model denoted as METMODEL.

The METMODEL is based on a simple assumption that fluctuations in energy measurements of jets, soft unclustered particles from the underlying event, and multiple interactions are the dominant sources of fake $E_{T}$. Therefore, the individual contributions of each of these components to fake $E_{T}$ can be modeled, on average, by smearing their energies according to the corresponding energy resolution functions. Jets are the dominant source of fake $E_{T}$ because they are collimated sprays of energetic particles in a certain direction and may have large measurement fluctuations in that direction. The unclustered energy, on the other hand, tends to be uniformly spread in the calorimeter. Therefore, the portion of $E_{T}$ due to this source is usually small and mostly results in a smearing of the jet component of fake $E_{T}$. Taking into account the above considerations and for reasons of simplicity, we model only the fake $E_{T}$ due to mismeasurements of jets and all soft unclustered energy (rather than individual unclustered particles).

The $E_{T}$ resolution due to the soft unclustered energy is studied in the $\gamma \gamma$ control sample (see Appendix A 2) and $Z / \gamma^{*} \rightarrow e^{+} e^{-}$events with $85 \mathrm{GeV} / c^{2}<M_{\mathrm{ee}}<$ $97.5 \mathrm{GeV} / c^{2}$. We fit distributions of $x$ and $y$ components of the $E_{T}$ for events without jets, $N_{\text {jet }}\left(E_{T}>15 \mathrm{GeV}\right)=0$, in small bins of $\sqrt{\sum E_{T}}$, with a sum of two Gaussian distributions. We assume that both Gaussian distributions have the same mean, but different widths $(\sigma$ and scale $\times \sigma$, respectively). From the individual fits of $E_{T}^{x}$ and $E_{T}^{y}$ distributions, we obtain the mean, $\sigma$, scale, and relative normalization, Norm, of two Gaussians for each bin of $\sqrt{\sum E_{T}}$ (bin size is $2 \mathrm{GeV}^{1 / 2}$ ). The parameters are then fitted by simple polynomial functions of $z=\sqrt{\sum E_{T}}: p_{0}+p_{1} z$ for $\sigma$ and the scale, $p_{0}+p_{1} z^{2}$ for the mean, and Norm $=p_{0}$. These functions provide a parameterization of the unclustered energy contribution into the $x$ and $y$ components of the fake $E_{T}$ in the event. The default set of parameters is obtained from the $\gamma \gamma$ control sample. We also use the results of fits in the data $Z \rightarrow e^{+} e^{-}$sample as an alternative set of parameters to study the associated systematic uncertainties. Figure 14 demonstrates an example of the $E_{T}^{y}$ resolution parameterization due to the unclustered energy
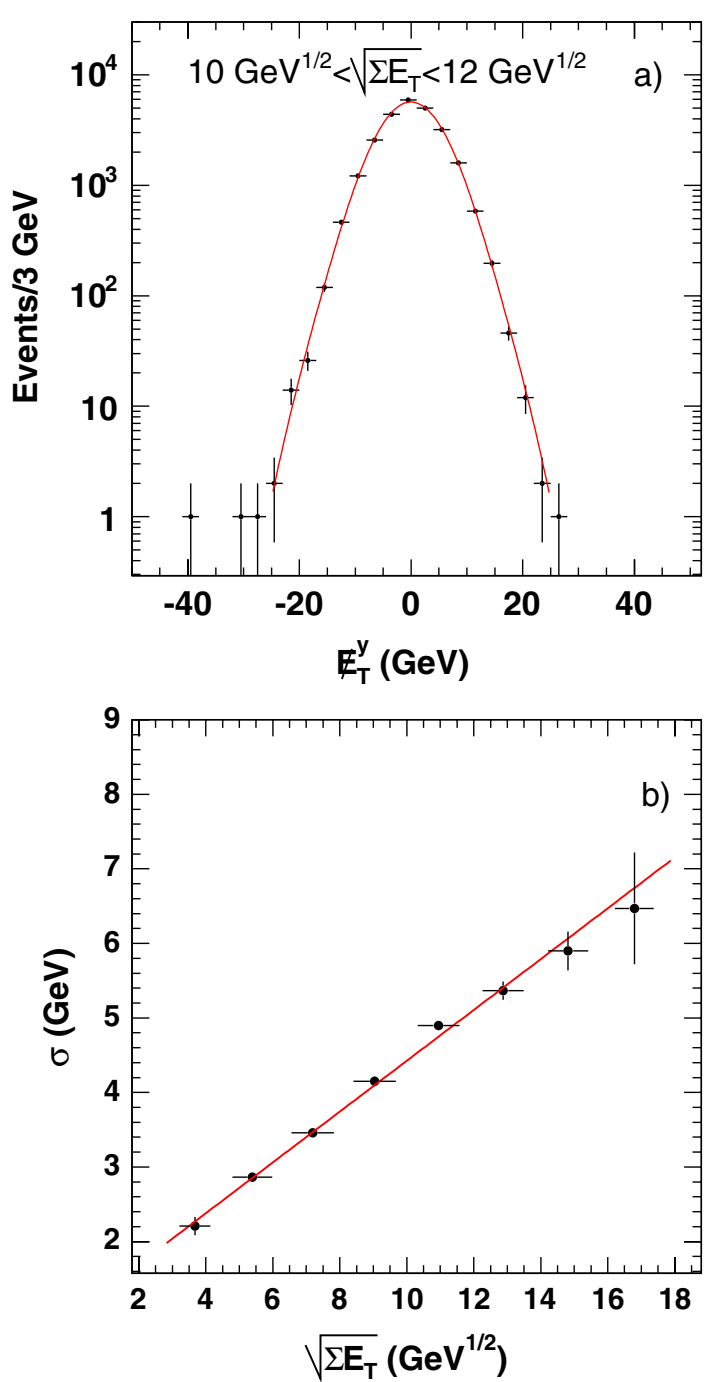

FIG. 14 (color online). Example of the fake $E_{T}$ parameterization due to unclustered energy. (a) two-Gaussian fit of the $E_{T}^{y}$ distribution for PYTHIA $\gamma \gamma$ events from one of the bins in $\sqrt{\sum E_{T}}$. (b) dependence of a width, $\sigma$, of the leading Gaussian depends on the $\sqrt{\sum E_{T}}$. On both plots, points are PYTHIA data and curves are the fit functions. 
in PYTHIA $\gamma \gamma$ events. Distributions for both $x$ and $y$ components of $E_{T}$ look essentially identical to those shown in Fig. 14. We also do not observe any large difference in the parameterization of the $E_{T}$ resolution due to unclustered energy between $Z / \gamma^{*} \rightarrow e^{+} e^{-}$and loose $\gamma \gamma$ events in data as well as between data and MC.

To account for contributions from jets into the fake $E_{T}$, we obtain the jet energy resolution, JER, as a function of jet energy and pseudorapidity, $E$ and $\eta$. For this purpose, we use PYTHIA samples of dijet and $Z$ - jet events passed through the GEANT-based detector simulation. In these events, we reconstruct jets before (hadron jet) and after (detector jet) the detector simulation by using the same cone clustering algorithm at both levels. The jet energy resolution is then defined as a ratio of the detector $\left(E^{\mathrm{det}}\right)$ and hadron level $\left(E^{\text {had }}\right)$ jet energies, $J E R=E^{\text {det }} / E^{\text {had }}-1$, for hadron and detector jets with $p_{T}>3 \mathrm{GeV} / c$ that are matched within a cone of $R(\phi, \eta)<0.1$. Unlike the energy balance in dijet and $Z$-jet events, this definition of JER is mostly sensitive to detector effects and allows us to significantly minimize the dependence of resolution on the effects of initial- and final-state radiation. However, we still compare the dijet and $Z$-jet balance in data and $\mathrm{MC}$ to make sure that the simulation adequately describes the resolution. We fill JER histograms for jets in $5 \mathrm{GeV}$ bins in jet energy and $\Delta \eta=0.2$ bins in pseudorapidity. We fit these histograms by a linear combination of Gaussian and Landau functions of $x$, where $x=-\mathrm{JER} /(1+\mathrm{JER})$ ensures stable fits in the entire range of jet energies. Examples of fits for one particular $\eta$ bin can be found in Fig. 15. These plots illustrate that our fit function successfully describes the jet energy resolution in a wide range of jet energies. It is also important to mention that the same fit function is used for all $\eta$ bins. From the individual fits for each $\left(E^{\text {jet }}, \eta\right)$ bin, we obtain a relative normalization, $C$, and parameters of a Gaussian (mean and $\sigma$ ) and Landau (mean and $\sigma$ ) fits. These parameters are plotted as a function of $E^{\text {jet }}$ for each $\eta$ bin, and fit with the following functions: $\sigma=\sqrt{p_{0} / E+p_{1}}$; mean $=p_{0}+p_{1} E+p_{2} / E$; and $C=\left(p_{0}+p_{1} \sqrt{E}\right) / E+p_{2}$. This provides a smooth parameterization of JER for all reconstructed jets with $E^{\text {jet }}>3 \mathrm{GeV}$ and $|\eta|<3.6$.

We predict the shape of fake $E_{T}$ based on the energy resolution functions described above. For each event, we produce a probability distribution function, $\mathcal{P}\left(E_{T}\right)$, of all possible values of the fake $E_{T}$ by smearing the energies of jets and unclustered energy according to these objects' individual resolution functions in a large number of pseudoexperiments. Then, we sum up these individual $\mathcal{P}\left(E_{T}\right)$ distributions for all events to obtain a shape of the predicted fake $E_{T}$ due to energy mismeasurements in our data sample. Technical details of how we generate $\mathcal{P}\left(E_{T}\right)$ are given below. An example of this $\mathcal{P}\left(E_{T}\right)$ distribution for one of the $\gamma \gamma$ baseline sample events can be found in Fig. 16. The method is validated in MC samples with and without
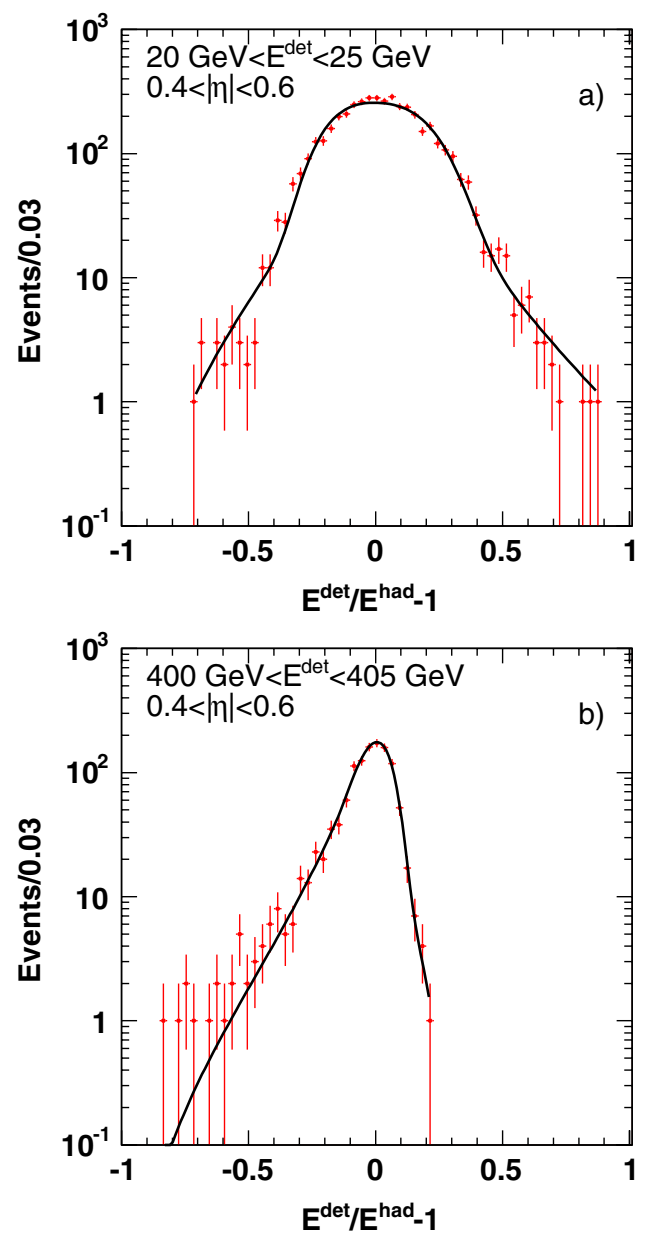

FIG. 15 (color online). Examples of jet energy resolutionfits using a linear combination of Gaussian and Landau functions of $x=-\mathrm{JER} /(1+\mathrm{JER})$ where $\mathrm{JER}=E^{\mathrm{det}} / E^{\text {had }}-1$ for two different jet energy bins: (a) $20 \mathrm{GeV}<E^{\text {det }}<25 \mathrm{GeV}$ and (b) $400 \mathrm{GeV}<E^{\mathrm{det}}<405 \mathrm{GeV}$.

intrinsic $E_{T}$. Figure 17 demonstrates that the METMODEL successfully predicts the shape of $E_{T}$ distributions in PYTHIA $\gamma \gamma$ and PYTHIA $Z \rightarrow e^{+} e^{-}$events with fake $E_{T}$. The technique is also cross-checked by performing the entire analysis with the data $\gamma \gamma$ control sample and data $Z \rightarrow e^{+} e^{-}$sample.

The $\mathcal{P}\left(E_{T}\right)$ distribution for a given event can be obtained using a large number of pseudoexperiments. For each pseudoexperiment, we start by forming a list of all jets with $E_{T}>3 \mathrm{GeV}$ and $|\eta|<3.0$ in this event and then smear their energies according to $\operatorname{JER}\left(E^{\mathrm{jet}}, \eta\right)$ described above. If the smeared jet energy, $E_{T}^{\text {smear }}$, is above the $15 \mathrm{GeV}$ threshold, we calculate the contribution of that jet into the fake $E_{T}: \vec{E}_{T}^{\mathrm{jet}, i}=\vec{E}_{T}-\vec{E}_{T}^{\text {smear }}$. Therefore, the METMODEL should account for a correlation between the directions of $E_{T}$ and jets. Then, we recalculate the unclustered energy based on $E_{T}^{\text {smear }}$ of all jets to avoid double-counting when one of the jets with $E_{T}<15 \mathrm{GeV}$ has $E_{T}^{\text {smear }}>15 \mathrm{GeV}$. For the next step, we randomly generate the expected $x$ and $y$ components of the $E_{T}$ contribution due to the unclustered energy 

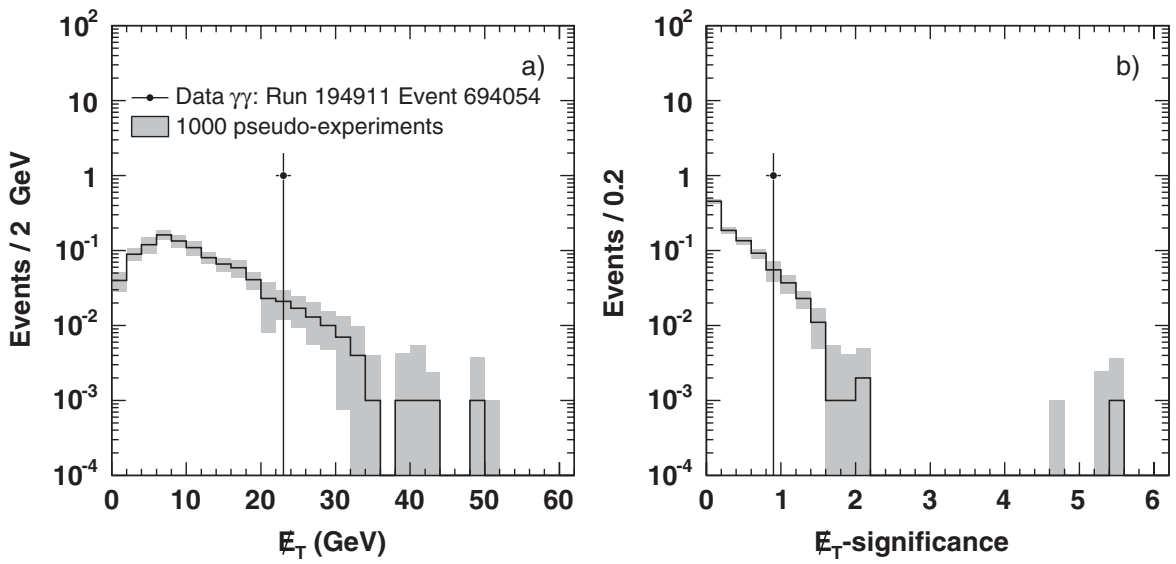

FIG. 16. Examples of the generated (a) $\mathcal{P}\left(E_{T}\right)$ and (b) $E_{T}$-significance distributions for one of the signal sample events.

deposited in the calorimeter. This procedure also accounts, on average, for effects of energy resolution of photons and electrons as well as residual effects of the wrong vertex choice. Finally, we take a vector sum of all individual $E_{T}$ components due to the soft unclustered energy and each of the jets with $E_{T}^{\text {smear }}>15 \mathrm{GeV}$ to obtain the final prediction of the fake $E_{T}$.

The METMODEL is not designed to predict the exact value of the fake $E_{T}$ in each event. Instead, it provides a twodimensional probability density function, $\mathcal{P}\left(\mathbb{E}_{T}\right)$, for values of the fake $E_{T}$ which could arise from energy mismeasurements in the calorimeter. This $\mathcal{P}\left(\vec{E}_{T}\right)$ can be used to determine a significance of the observed $E_{T}$ in a given event according to the following formula:

$$
E_{T} \text {-significance }=-\log _{10}\left(1-\int_{0}^{\vec{w}} \mathcal{P}(\vec{z}) d \vec{z}\right)
$$

where $\vec{z}$ is the generated fake $\vec{E}_{T}$ and $\vec{w}$ is the observed $\vec{E}_{T}$. The $E_{T}$-significance defined by Eq. (C1) correctly takes into account all of the correlations between jets and the observed $E_{T}$. However, the method has one significant drawback since it requires generating a large number of pseudoexperiments (e.g., $>10^{6}$ pseudoexperiments for $E_{T}$-significance $=6$ ). To overcome this problem, we take a simplified path of calculating an upper limit on the $E_{T}$-significance ("raw" $E_{T}$-significance) according to the formula

$E_{T}$-significance $=-\log _{10}\left(\tilde{\mathcal{P}}_{\text {jets }} \tilde{\mathcal{P}}_{\text {uncl }}\right)$,

$$
\begin{aligned}
\tilde{\mathcal{P}}_{\text {uncl }} & =\prod_{i=x, y}\left(1-\int_{-u_{i}}^{u_{i}} \mathcal{P}_{\text {uncl }}^{i}(u) d u\right), \quad u_{i}=E_{T}{ }^{x}, E_{T}{ }^{y}, \\
\tilde{\mathcal{P}}_{\text {jets }} & =\prod_{i, v_{i}>0}^{\text {jets }} \int_{-1}^{v_{i}} \mathcal{P}_{i}(v) d v \times \prod_{i, v_{i}<0}^{\text {jets }}\left(1-\int_{-1}^{v_{i}} \mathcal{P}_{i}(v) d v\right) v_{i} \\
& =E_{T} /\left(E_{T}^{i} \cos \Delta \phi_{i}\right),
\end{aligned}
$$

where $\mathcal{P}_{\text {uncl }}^{x, y}(u)$ is the probability density function for unclustered energy contribution to $E_{T}$ resolution [illustrated in Fig. 14(a)], $\mathcal{P}_{i}(v)$ is the probability density function for jet energy resolution (shown in Fig. 15), $E_{T}^{i}$

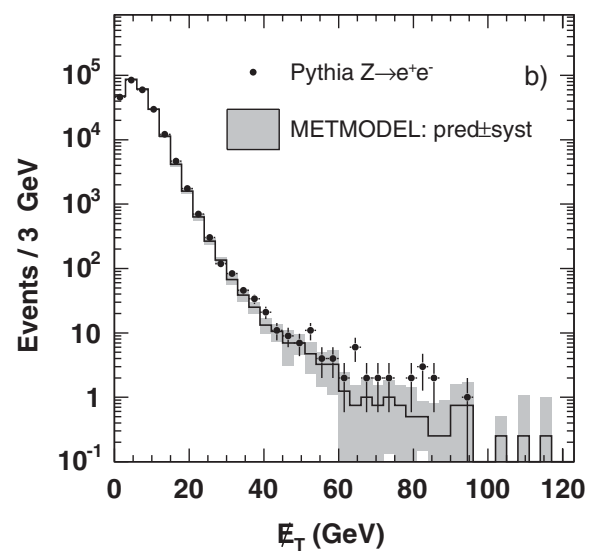

FIG. 17. Examples of the METMODEL predictions for $E_{T}$-distributions in PYTHIA (a) $\gamma \gamma$ and (b) $Z \rightarrow e^{+} e^{-}$events. These events do not have the intrinsic $E_{T}$. However, fluctuations in energy measurements can result in the fake $E_{T}$ as large as $100 \mathrm{GeV}$. Both distributions are well described by the METMODEL predictions in the entire range of the observed $E_{T}$. 
is the transverse energy of the $i$ th jet, and $\cos \Delta \phi_{i}$ is the azimuthal angle between that jet and measured $E_{T}$. The raw $E_{T}$-significance obtained from Eq. (C2) is then calibrated to have a simple shape defined a priori: $d N / d x=N_{\text {evnt }} \cdot \ln (10.0) \times 10^{-x}$, where $x$ is the $E_{T}$-significance and $N_{\text {evnt }}$ is the number of events in a sample. The shape of the $E_{T}$-significance has one important property: if all events in a data sample were to have only fake $E_{T}$, then $N_{\text {evnt }} \cdot 10^{- \text {cut }}$ events would pass a requirement $E_{T}$-significance $>$ cut. This property makes it very easy to calibrate the $E_{T}$-significance by means of pseudoexperiments. In each pseudoexperiment, we obtain a randomly generated value of $E_{T}$. Then we calculate the significance of this generated $E_{T}$ as if it were measured $E_{T}$. We repeat this procedure for all events in the data sample and obtain the significance distribution for pseudoexperiments. Finally, an adjustment factor is derived for each bin of the distribution so that the corrected $E_{T}$-significance satisfies the $N\left(E_{T}\right.$-significance $>$ cut $)=N_{\text {evnt }} \cdot 10^{- \text {cut }}$ requirement.

The systematic uncertainties associated with the METMODEL predictions are evaluated by comparing the results obtained with the default set of parameters to predictions obtained with the METMODEL parameters changed by 1 standard deviation $( \pm \sigma)$. In total, ten sources of the systematic uncertainties are considered: (1) difference in the unclustered energy parameterization of the $E_{T}$ resolution for $\gamma \gamma$ control and $Z \rightarrow e^{+} e^{-}$events; (2) uncertainties on four parameters of the unclustered energy parameterization; (3) uncertainties on five parameters of the JER parameterization. The correlations between these parameters are also taken into account. The statistical uncertainty that depends on the number of pseudoexperiments per event and the systematic uncertainty are added in quadrature to obtain the total uncertainty.
[1] S. Abe et al., arXiv:hep-ph/0003154; R. L. Culbertson et al., arXiv:hep-ph/0008070; B. Allanach et al., arXiv: hep-ph/9906224; S. Ambrosanio et al., arXiv:hep-ph/ 0006162; M. S. Carena et al., arXiv:hep-ph/0010338.

[2] S. L. Glashow, Nucl. Phys. 22, 579 (1961); S. Weinberg, Phys. Rev. Lett. 19, 1264 (1967); A. Salam, in Proceedings of the 8th Nobel Symposium N. Svartholm (Almqvist and Wiksell, Stockholm, 1968); D. J. Gross and F. Wilczek, Phys. Rev. D 8, 3633 (1973).

[3] The CDF II detector uses a cylindrical coordinate system in which $\phi$ is the azimuthal angle, $\theta$ is the polar angle with respect to the proton beam, $r$ is the radius from the nominal beam line, and $z$ points in the proton beam direction, with the origin at the center of the detector. The transverse $r-\phi$, or $x-y$ plane, is the plane perpendicular to the $z$ axis. The pseudorapidity, $\eta$, is defined as $-\ln (\tan (\theta / 2))$. The transverse energy of a particle is $E_{T}=E \cdot \sin (\theta)$. The transverse momentum of a particle is defined as $p_{T}=p \cdot \sin (\theta)$.

[4] S. Dimopoulos, S. D. Thomas, and J. D. Wells, Nucl. Phys. B488, 39 (1997); S. Ambrosanio, G. D. Kribs, and S.P. Martin, Phys. Rev. D 56, 1761 (1997); G. F. Giudice and R. Rattazzi, Phys. Rep. 322, 419 (1999); S. Ambrosanio, G. L. Kane, G. D. Kribs, S. P. Martin, and S. Mrenna, Phys. Rev. D 55, 1372 (1997).

[5] B. A. Dobrescu, Phys. Rev D 63, 015004 (2000); G. Landsberg and K. Matchev, Phys. Rev D 62, 035004 (2000).

[6] K.D. Lane and S. Mrenna, Phys. Rev D 67, 115011 (2003).

[7] G. Bhattacharyya and R. N. Mohapatra, Phys. Rev. D 54, R4204 (1996).

[8] L. Hall and C. Kolda, Phys. Lett. B 459, 213 (1999); M. C. Kumar, Prakash Mathews, V. Ravindran, and Anurag Tripathi, Phys. Lett. B 672, 45 (2009).
[9] F. Abe et al. (CDF Collaboration), Phys. Rev. Lett. 81, 1791 (1998); Phys. Rev. D 59, 092002 (1999).

[10] D. Acosta et al. (CDF Collaboration), Phys. Rev. D 71, 031104 (2005).

[11] V. M. Abazov et al. (D0 Collaboration), Phys. Lett. B 659, 856 (2008).

[12] A. Abulencia et al. (CDF Collaboration), Phys. Rev. D 75, 112001 (2007).

[13] T. Aaltonen et al. (CDF Collaboration), Phys. Rev. Lett. 99, 171801 (2007).

[14] T. Aaltonen et al. (CDF Collaboration), Phys. Rev. Lett. 103, 061803 (2009).

[15] T. Aaltonen et al. (CDF Collaboration), Phys. Rev. D 79, 011101 (2009).

[16] D. Acosta et al. (CDF Collaboration), Phys. Rev. D 71, 032001 (2005).

[17] C. S. Hill et al., Nucl. Instrum. Methods Phys. Res., Sect. A 511, 118 (2003).

[18] A. Sill et al., Nucl. Instrum. Methods Phys. Res., Sect. A 447, 1 (2000).

[19] A. Affolder et al., Nucl. Instrum. Methods Phys. Res., Sect. A 453, 84 (2000).

[20] A. Affolder et al., Nucl. Instrum. Methods Phys. Res., Sect. A 526, 249 (2004).

[21] L. Balka et al., Nucl. Instrum. Methods Phys. Res., Sect. A 267, 272 (1988); S. R. Hahn et al., ibid. 267, 351 (1988).

[22] S. Bertolucci et al., Nucl. Instrum. Methods Phys. Res., Sect. A 267, 301 (1988).

[23] R. Oishi et al., Nucl. Instrum. Methods Phys. Res., Sect. A 453, 227 (2000); M. Albrow et al., ibid. 480, 524 (2002).

[24] M. Goncharov et al., Nucl. Instrum. Methods Phys. Res., Sect. A 565, 543 (2006).

[25] G. Ascoli et al., Nucl. Instrum. Methods Phys. Res., Sect. A 268, 33 (1988); A. Artikov et al., Nucl. Instrum. Methods Phys. Res., Sect. A 538, 358 (2005). 
[26] D. Acosta et al., Nucl. Instrum. Methods Phys. Res., Sect. A 494, 57 (2002).

[27] F. Abe et al. (CDF Collaboration), Nucl. Instrum. Methods Phys. Res., Sect. A 271, 387 (1988).

[28] T. Stelzer and W.F. Long, Comput. Phys. Commun. 81, 357 (1994); F. Maltoni and T. Stelzer, J. High Energy Phys. 02 (2003) 027.

[29] T. Sjostrand, Comput. Phys. Commun. 82, 74 (1994); S. Mrenna, Comput. Phys. Commun. 101, 232 (1997).

[30] R. Brun et al., CERN Report No. CERN-DD/EE/84-1, 1987.

[31] D. Acosta et al. (CDF Collaboration), Phys. Rev. Lett. 94, 041803 (2005); H. S. Hayward, Ph.D. thesis, University of Liverpool, 2005; M.H. Kirby, Ph.D. thesis, Duke University, 2004.

[32] H. L. Lai et al., Eur. Phys. J. C 12, 375 (2000).

[33] T. Aaltonen et al. (CDF Collaboration), Phys. Rev. D 77, 052002 (2008); M. Griffiths, Ph.D. thesis, University of Liverpool, 2007.

[34] D. Acosta et al. (CDF Collaboration), Phys. Rev. D 71, 051104 (2005).

[35] The vertex misassignment mostly happens in real $\gamma \gamma$ events. However, it also occurs in $\gamma \gamma_{\text {fake }}$ or $\gamma_{\text {fake }} \gamma_{\text {fake }}$ events, but at a much lower rate because these events tend to have more tracks associated with a vertex produced by the hard primary interaction vertex.

[36] The $W \gamma$ and $Z \gamma$ events are simulated using the leadingorder (LO) event generator [37]. The initial-state radiation (resulting in additional jets or photons), underlying event, and additional interactions in the same bunch crossing are modeled with the PYTHIA [29] Monte Carlo program. The generated events are processed through a realistic GEANTbased [30] detector simulation.

[37] U. Baur, T. Han, and J. Ohnemus, Phys. Rev. D 48, 5140 (1993); J. Ohnemus, Phys. Rev. D 47, 940 (1993).

[38] Sometimes a photon or an energetic $\pi^{0} / \eta^{0}$ from a jet can hit the area very close to the calorimeter cracks at $\eta \sim 0$ and $|\eta| \sim 1$.1. In such cases, these objects can get reconstructed as narrow jets with just a few towers $(<10)$ and a few tracks $(<5)$ pointing to them. Often, most of the energy $(\sim 85 \%)$ of these narrow jets is deposited either in the electromagnetic or hadronic calorimeters.

[39] The inclusive $W$ and $Z / \gamma^{*}$ events are simulated using the PYTHIA [29] Monte Carlo program and processed through a realistic GEANT-based [30] detector simulation.

[40] T. Aaltonen et al. (CDF Collaboration), Phys. Rev. D 78, 032015 (2008).

[41] T. Aaltonen et al. (CDF Collaboration), Phys. Rev. Lett. 103, 091803 (2009).

[42] T. Aaltonen et al. (CDF Collaboration), Phys. Rev. Lett. 104, 011801 (2010).

[43] For the central calorimeter, the fiducial region covers $\sim 87 \%$ of the total area.

[44] When counting central electromagnetic towers, we exclude towers associated with photon candidates. To reduce the rate at which real collision events are misidentified as beam halo, the total transverse energy of all contributing towers has to be less than $0.6 \mathrm{GeV}$.

[45] In this analysis, the EM timing is calculated with respect to the nominal center of the detector, $z=0 \mathrm{~cm}$. This leads to a larger resolution compared to the case when the actual interaction position, $z_{\mathrm{vertex}}$, and time, $t_{\mathrm{vertex}}$, are taken into account [24].

[46] A. Abulencia et al. (CDF Collaboration), J. Phys. G 34, 2457 (2007); Phys. Rev. D 74, 072006 (2006).

[47] A. Abulencia et al. (CDF Collaboration), Phys. Rev. D 75, 092004 (2007).

[48] F. Abe et al. (CDF Collaboration), Phys. Rev. D 45, 1448 (1992).

[49] A. Bhatti et al., Nucl. Instrum. Methods Phys. Res., Sect. A 566, 375 (2006).

[50] T. Aaltonen et al. (CDF Collaboration), Phys. Rev. D 78, 052006 (2008). 